\title{
Computer Aided Design of Geodesic Forms
}

\author{
Dimitra Tzourmakliotou \\ Laboratory of Steel Structures, Department of Civil Engineering, Democritus University of Thrace, Greece
}

\begin{abstract}
Geodesic forms constitute an important family of structural systems. They are efficient and appealing and are employed frequently for dome structures. Data generation for geodesic forms has always been a difficult task, from the days of hand data generation using tables of coefficients to today's specially written computer programs with many limitations and shortcomings. Formex algebra is a mathematical system which is ideally suited for data generation and configuration processing. The objective of the present paper is to introduce the concepts of formex algebra through which data generation for polyhedric and geodesic forms can be handled with ease and elegance.
\end{abstract}

Key words: Geodesic Form, formex algebra polyhedra, structural configuration, retronorm.

\section{Introduction}

Formex algebra is a mathematical system that provides a convenient basis for solution of problems in data generation and computer graphics. The ideas are general and have applications in many branches of science and technology. In particular, the concepts are suitable for use in computer aided analysis and design of geodesic forms and it is this area of application that will be the focus of attention in this paper. In presenting the material the emphasis is on the manner in which the concepts of formex algebra are employed rather than the details of the formulations and no prior knowledge of formex algebra is necessary for following the material [1]. The paper does contain some formex formulations but these are included to give a feel for the appearance of formex formulations rather than their details being essential for understanding of the material.

A geodesic dome is obtained from projecting the triangular faces of a regular or semiregular polyhedron on a surface such as a sphere, ellipsoid, etc. The geodesic domes have been developed by the American designer Buckminster Fuller, who 60 years ago turned

Corresponding author: Dimitra Tzourmakliotou, PhD, assistant professor, research fields: steel structures, space structures. E-mail: dimitratz@gmail.com. the architect's attention to the advantages of skeleton domes [2]. In this type of dome, the bracing members lie on the great circles of a sphere, thus following the geodesic lines of the surface. The geometry of the geodesic domes is based either on regular or semi-regular polyhedra. The faces of the polyhedra of this group are equal regular polygons of several classes. They occur everywhere in nature, in one form or another. For example a crystal of common salt is a cube and the crystalline form of carbon called a diamond, is octahedral.

The paper in this paper the generation of geodesic forms is solved in two stages. Firstly, a function called the "polyhedron function" is employed [3]. The resulting configuration is referred to as a "polyhedric configuration". The polyhedron function constitutes the kernel of the problem handling strategy for the data generation of geodesic forms. In the next stage, the "tractation retronorm" is employed to obtain the projection of the polyhedric configuration on one or more specified surfaces. Different types of projection such as central axial or parallel may also be used to generate interesting geodesic forms.

As mentioned above the first stage in the generation of a geodesic form is the creation of the polyhedric configuration. The approach presented in the paper provides a methodology that allows polyhedric 
configuration of all kinds to be generated in a convenient manner. However, polyhedric configurations based on regular polyhedra (known as Platonic solids) or semiregular (Archimedean) polyhedra are used to demonstrate the concepts and constructs through which polyhedric configurations may be created.

\section{Platonic and Archimedean Polyhedra}

During A "regular polyhedron" (known as Platonic solid) is a polyhedron whose faces are congruent regular polygons [4]. There are only five regular polyhedra, these are:
(1) Tetrahedron
(4 triangular faces)
(2) Cube (Hexahedron)
(6 square faces)
(3) Octahedron
(8 triangular faces)
(4) Dodecahedron
(12 pentagonal faces)
(5) Icosahedron
(20 triangular faces)

and are as shown in (Figs. 1-5).

Fig. 1 Tetrahedron.
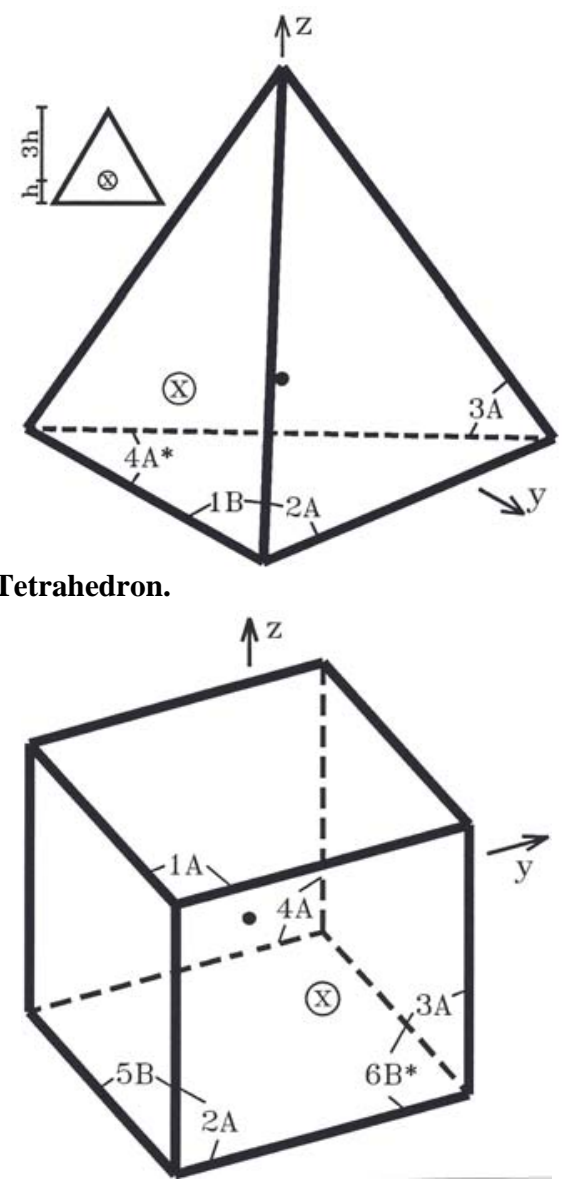

Fig. 2 Hexahedron (Cube).

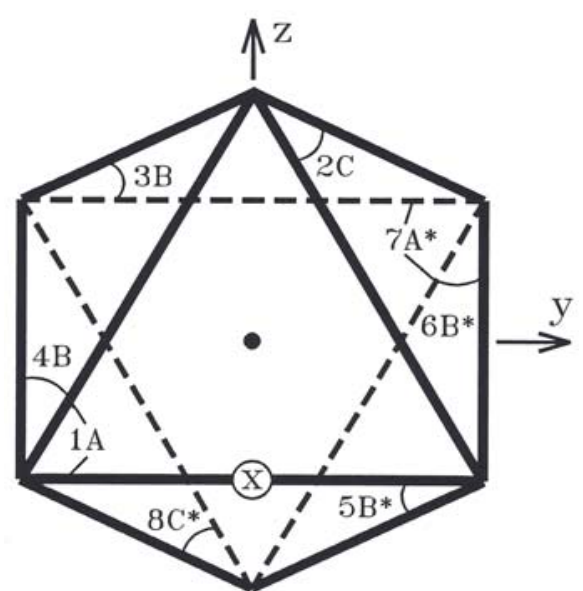

Fig. 3 Octahedron.

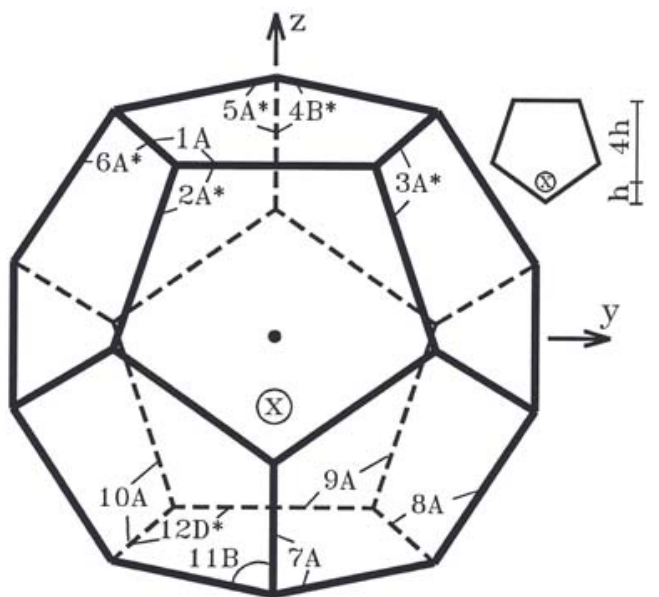

Fig. 4 Dodecahedron.

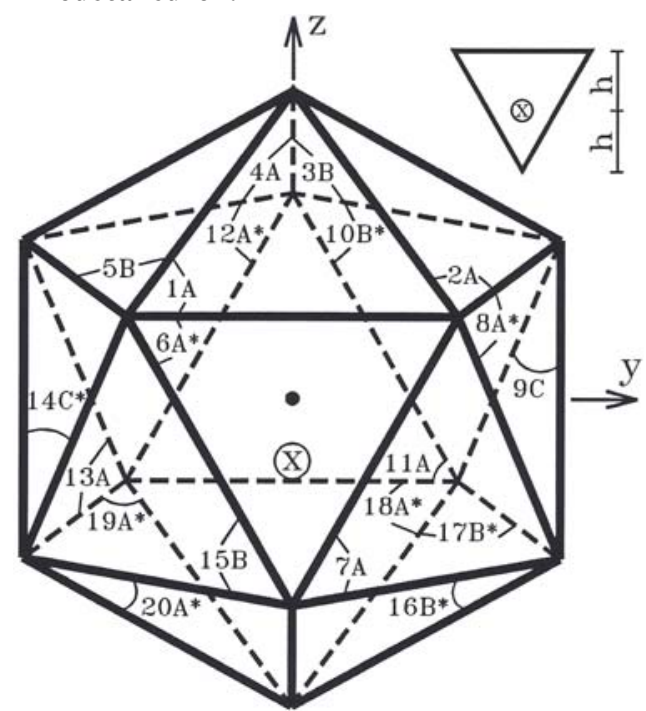

Fig. 5 Icosahedron.

The Platonic solid were discovered by Plato, but they have been so named because of their appearance in the Timaues [5]. In Timaues, Plato identifies four of the regular polyhedra with the four elements, the 
tetrahedron represents fire; the cube earth; the octahedron, air and the icosahedron water. The fifth and the last regular polyhedron the dodecahedron represents the quintessence of which the heavenly bodies are made and in which the four elements are impregnated. Each Platonic polyhedron can be surrounded by a sphere so that all its vertices touch the sphere. The sphere which circumscribes a Platonic polyhedron is referred to as the "circumscribing sphere" or "circumsphere" of the polyhedron (Fig. 6). The centre of the polyhedron is the same as the centre of its circumsphere.

Each face of the polyhedron is identified with a "face code" which is given at one corner of the face. A face code consists of a number followed by a letter and possibly followed by an asterisk. The number in a face code is the identification number of the face. The letter in a face code determines the points A, B, C,..., etc of the configuration that is to be placed on the indicated corner of the face. These letters for configurations corresponding to different shapes of polyhedral faces are as shown in Fig. 7. If a face code has an asterisk, it implies that the configuration which is to be placed on the face is the reflection, with respect the $x-y$ plane, of the given configuration.

The Archimedean or "semiregular polyhedra" are what is called "facially" regular polyhedra. This means that every face is a regular polygon though the faces are not all of the same kind. There are thirteen semiregular polyhedra which were discovered in ancient Greece and were described by Archimedes thus they are

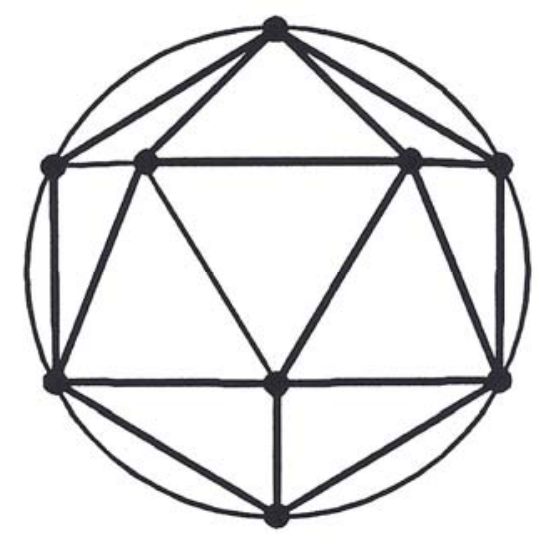

Fig. 6 Circumscribing sphere of the icosahedron

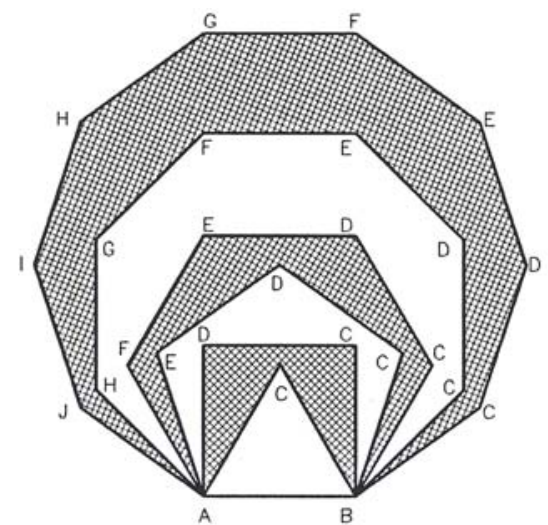

Fig. 7 Different shapes of polyhedral faces.

called the Archimedean polyhedra. The Archimedean polyhedra are given in Table 2 and are shown in Figs. 8-20.

These polyhedra consist of various combinations of triangles, squares, pentagons, hexagons, octagons and decagons (Fig. 7). Ten of the Archimedean polyhedra utilize only two kinds of polygons and the remaining three utilize three kinds of polygons, as shown in Table 1. Like in a Platonic polyhedron an Archimedean polyhedron has a circumsphere touching all of its vertices. These figures should be interpreter in a way similar to the one given for the Platonic Polyhedra. The significance of the number, the letter and the asterisk which are given for each one of the faces of an Archimedean polyhedron in the figures is as explained before.

\section{Polyhedron Function}

Sustainable consider a polyhedron and let some given configurations be placed on its faces. The result is referred to as a "polyhedric configuration" or "polyhedric form". This term may also be used to refer to a portion of a polyhedric configuration. A polyhedron which is used as the basis for the creation of a polyhedric configuration or form is referred to as the "base polyhedron" of the polyhedric form. This is a formex function called "polyhedron function" that can be used to crate formices representing polyhedric configurations [6-7]. The applications of the polyhedron function may be described with the help of an example. 


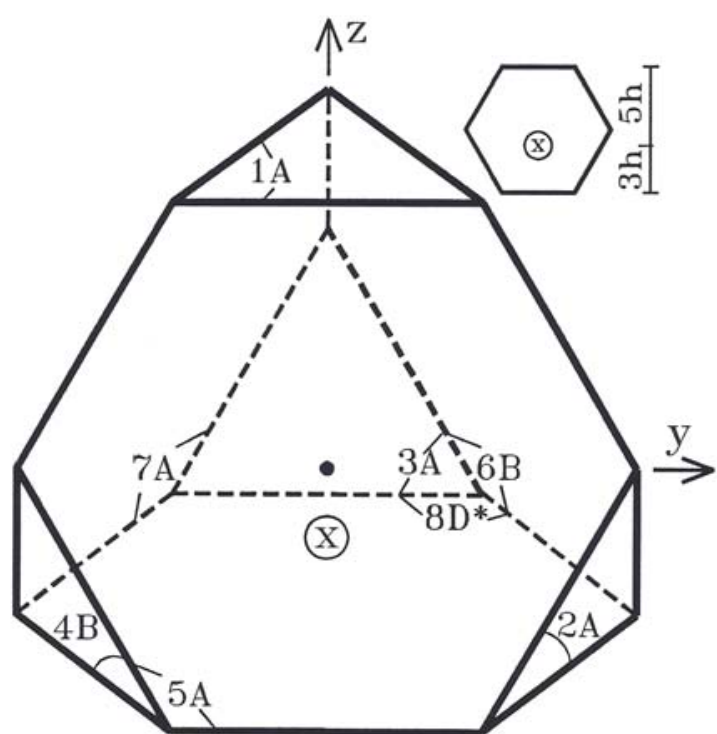

Fig. 8 Truncated tetrahedron.

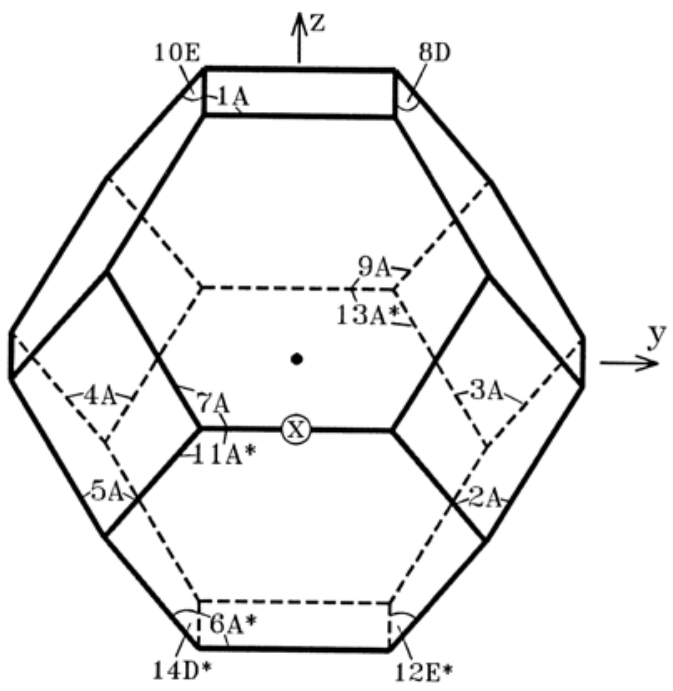

Fig. 9 Cuboctehedron.

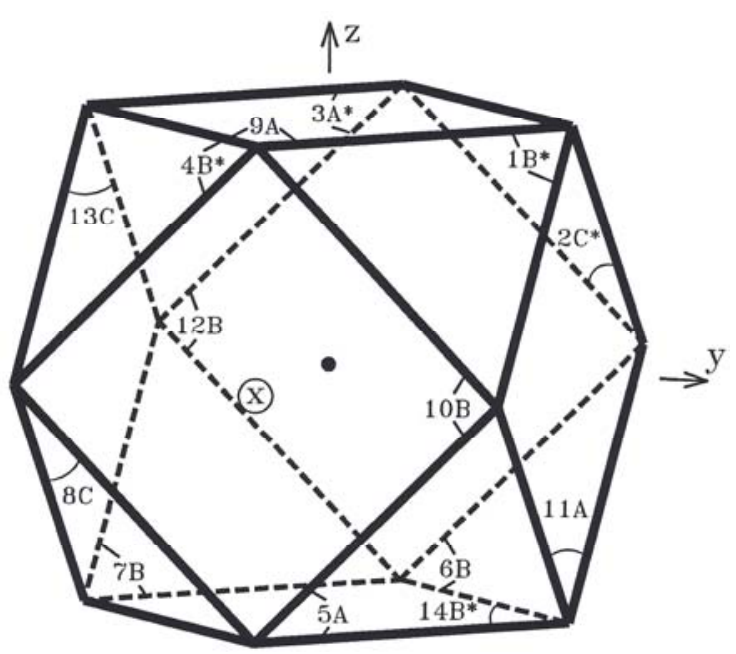

Fig. 10 Truncated cube.

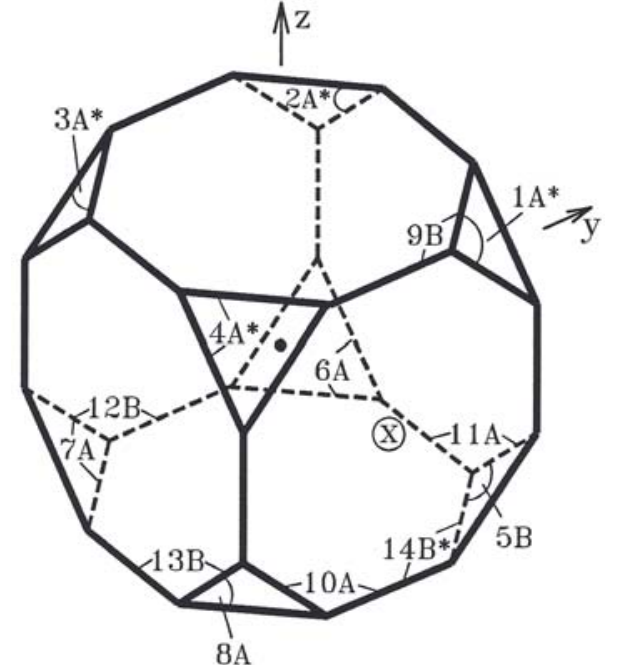

Fig. 11 Truncated octahedron.

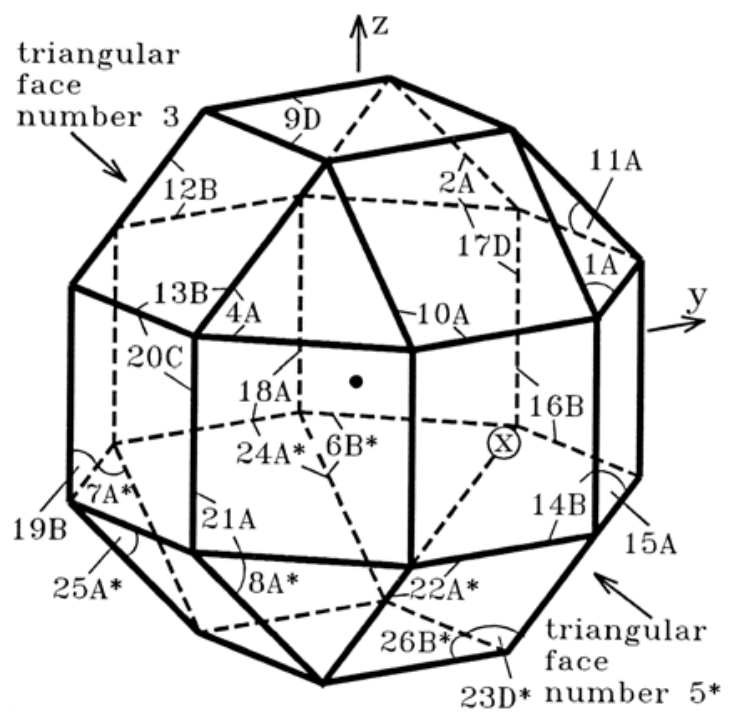

Fig. 12 Small rhombicuboctahedron.

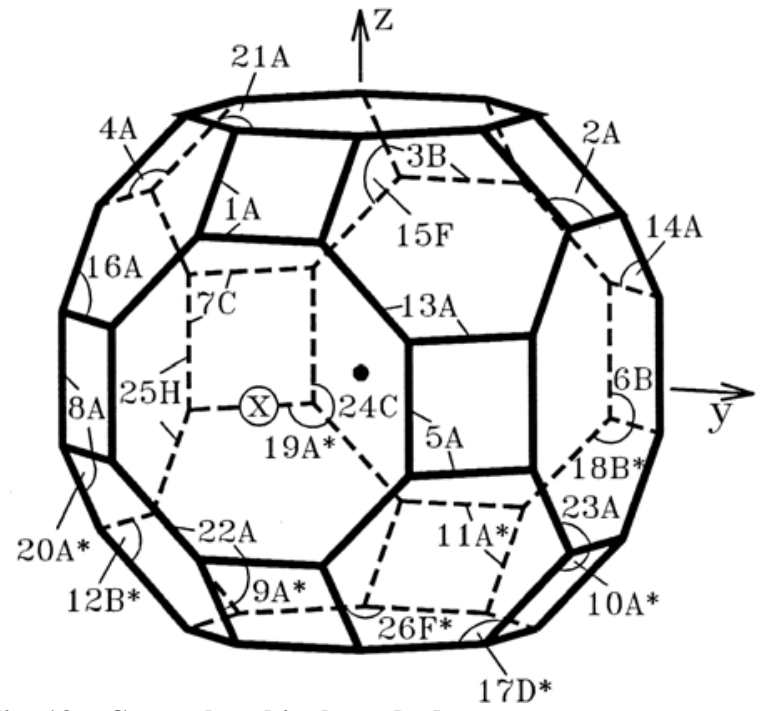

Fig. 13 Great rhombicuboctahedron. 


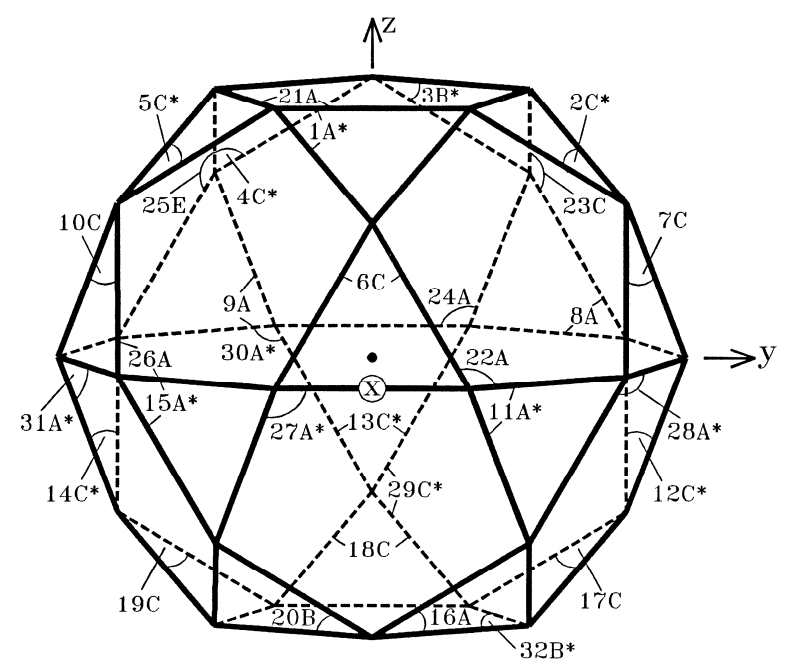

Fig. 14 Icosidodecahedron.

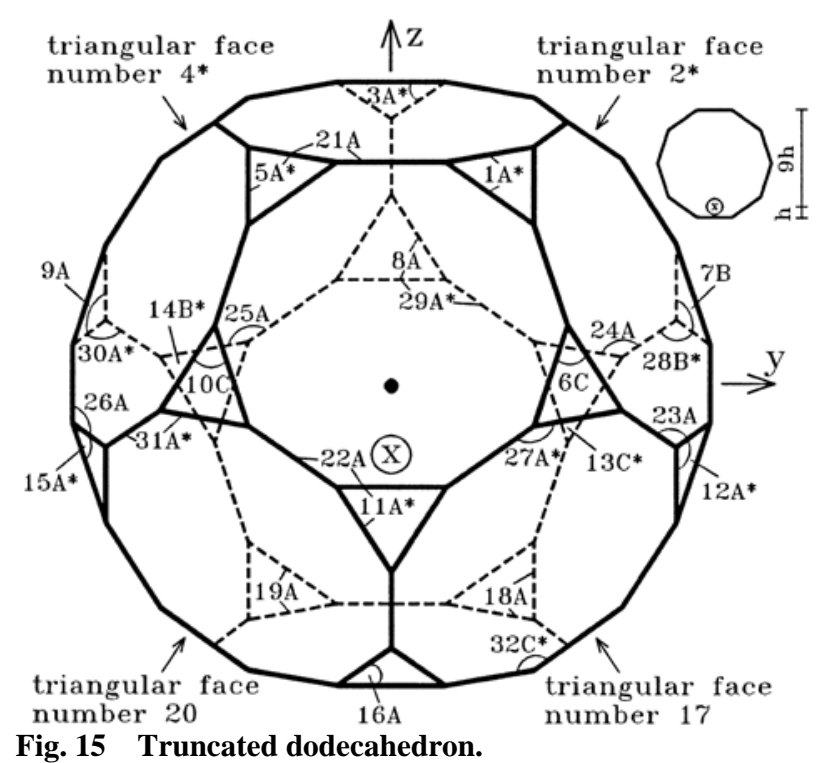

Fig. 15 Truncated dodecahedron.

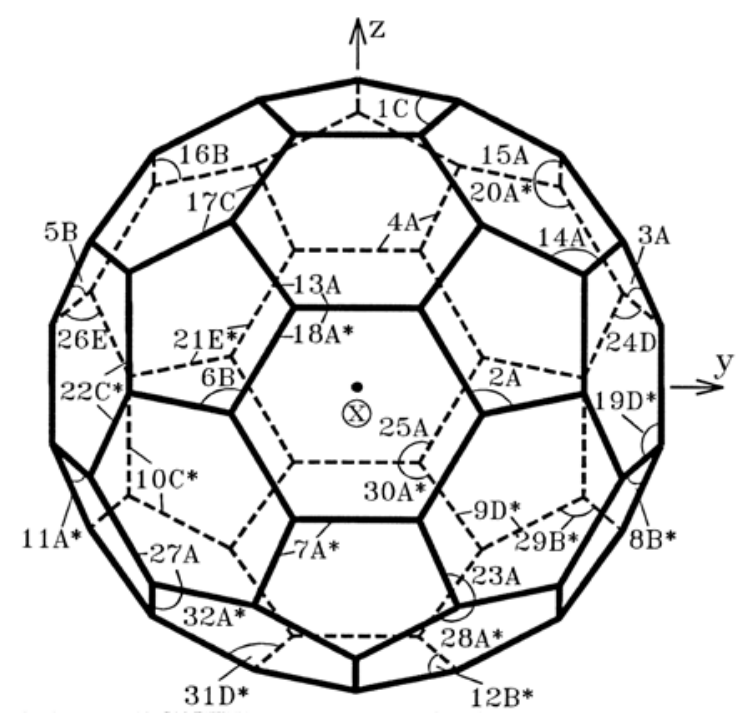

Fig. 16 Truncated icosahedron.

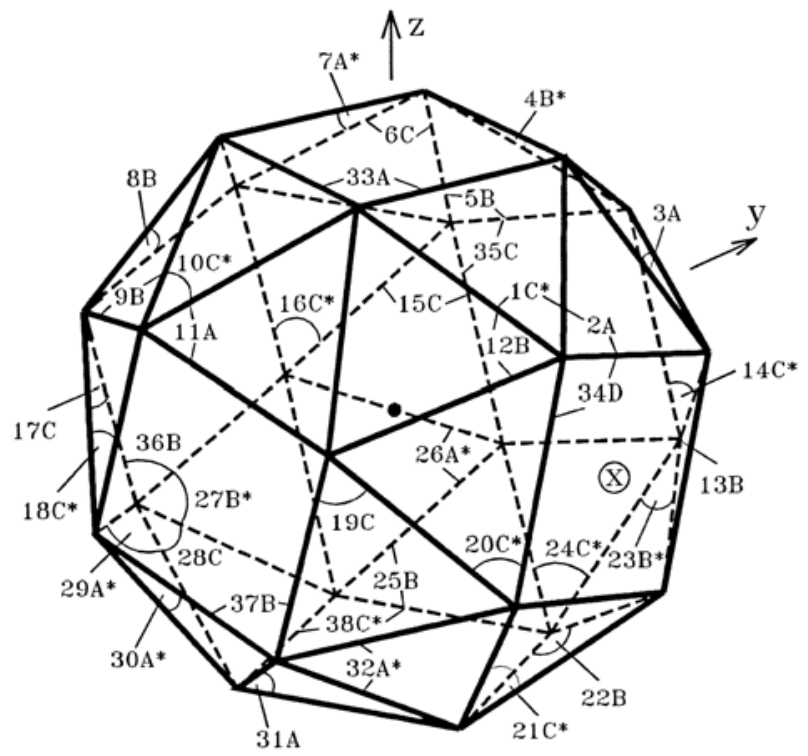

Fig. 17 Snub cube.

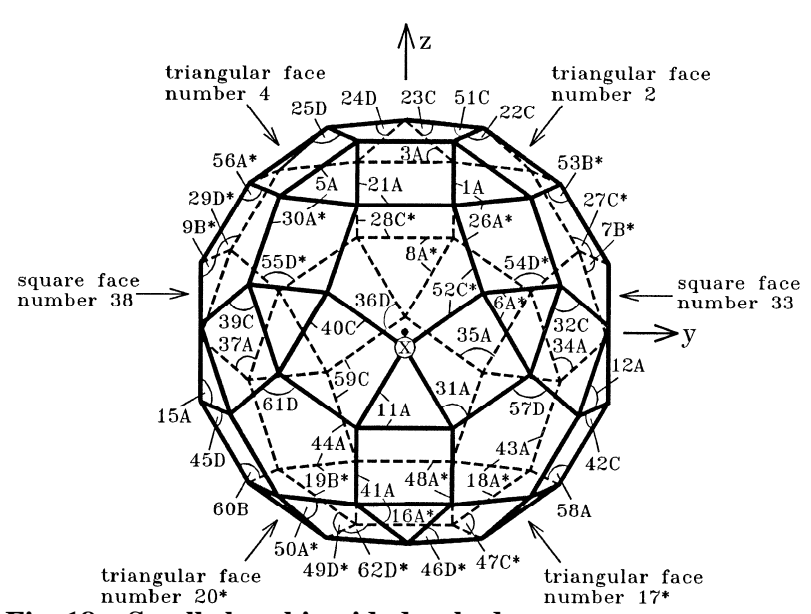

Fig. 18 Small rhombicosidodecahedron.

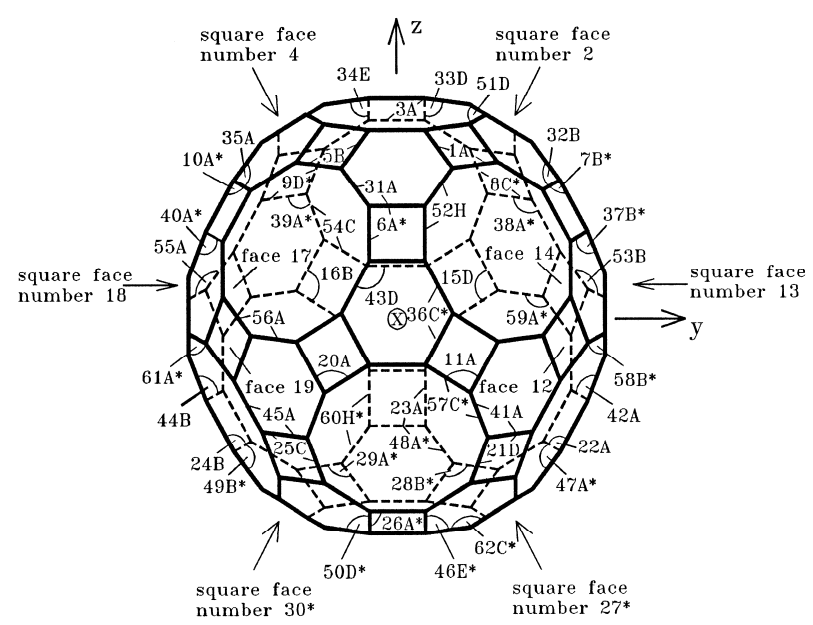

Fig. 19 Great rhombicosidodecahedron. 


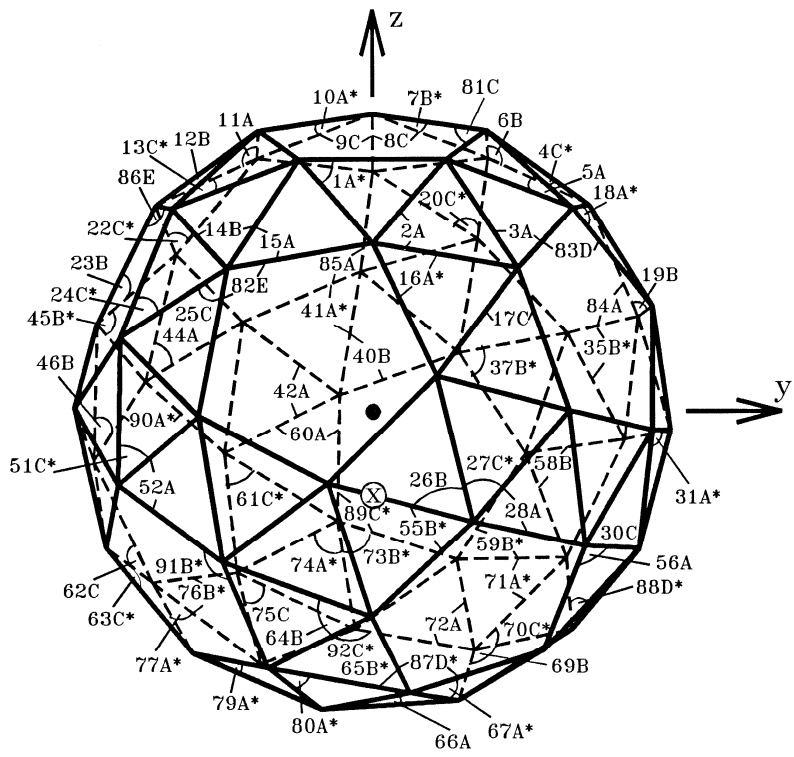

Fig. 20 Snub dodecahedron.

Table 1 The type of polygons in each of the archimedean polyhedra.

\begin{tabular}{|c|c|}
\hline Polyhedron & Type and number of faces \\
\hline Truncated tetrahedron & \\
\hline Cuboctehedron & 6 \\
\hline Truncated cube & \\
\hline Truncated octahedron & \\
\hline $\begin{array}{l}\text { Small } \\
\text { Rhombicuboctehedron }\end{array}$ & 18 \\
\hline $\begin{array}{l}\text { Great } \\
\text { rhombicuboctehedron }\end{array}$ & 12 \\
\hline Icosidodecahedron & \\
\hline Truncated dodecahedron & \\
\hline Truncated icosahedron & \\
\hline Snub cube & 32 \\
\hline $\begin{array}{l}\text { Small rhombi } \\
\text { icosidodecahedron }\end{array}$ & 30 \\
\hline $\begin{array}{l}\text { Great rhombi } \\
\text { icosidodecahedron }\end{array}$ & 30 \\
\hline Snub dodecahedron & $\angle>80$ \\
\hline
\end{tabular}

Table 2 The platonic and the archimedean polyhedra and their polyhedron code.

\begin{tabular}{|c|c|}
\hline Polyhedron & Code \\
\hline \multicolumn{2}{|l|}{ Platonic polyhedron } \\
\hline Tetrahedron & 1 \\
\hline Cube (Hexahedron) & 2 \\
\hline Octahedron & 3 \\
\hline Dodecahedron & 4 \\
\hline Icosahedron & 5 \\
\hline \multicolumn{2}{|l|}{ Archimedean polyhedra } \\
\hline Truncated tetrahedron & 6 \\
\hline Cuboctehedron & 7 \\
\hline Truncated cube & 8 \\
\hline Truncated octahedron & 9 \\
\hline Small rhombicuboctahedron & 10 \\
\hline Great rhombicuboctahedron & 11 \\
\hline Icosidodecahedron & 12 \\
\hline Truncated Dodecahedron & 13 \\
\hline Truncated icosahedron & 14 \\
\hline Snub cube (dextro) & 15 \\
\hline Snub cube (laevo) & -15 \\
\hline Small rhombicosidodecahedron & 16 \\
\hline Great rhombicosidodecahedron & 17 \\
\hline Snub dodecahedron (dextro) & 18 \\
\hline Snub dodecahedron (laevo) & -18 \\
\hline
\end{tabular}

Consider a single layer triangular configuration which will be referred to in the sequel as the configuration. The configuration together with the normat U1-U2-U3 for the formex formulation are shown in Fig. 21.

This configuration may be represented in terms of the formex variable $\mathrm{E}$ which is given as, $\mathrm{E}=\mathrm{LIB}(\mathrm{I}=0,5)|\mathrm{RIN}(1,6-\mathrm{I}, 2)| \operatorname{TRANID}(\mathrm{I}, \mathrm{I}) \mid\{[0,0 ; 2,0],[2$, $0 ; 1,1],[1,1 ; 0,0]\}$.

Let it be required to map this configuration onto all the faces of a tetrahedron. A Formian statement describing this operation may be given as shown in Fig. 22 where

$$
\mathrm{G}=\mathrm{BB}(1, \mathrm{TAN} \mid 60) \mid \mathrm{E}
$$

As has been stated in section 2 a tetrahedron has four equilateral triangular faces. The triangular configuration that is described here by formex $\mathrm{E}$ is not 
equilateral. Therefore, it will be necessary to scale the configuration using the appropriate scale factors. Formex variable $G$ specifies the scale factors that are used in the first and second direction to obtain the equilateral triangular configuration of Fig. 23. Formex variable $G$ represents the actual node coordinates while formex variable $\mathrm{E}$ represents the corresponding normat coordinates of the configuration. A graphical representation of formex variable $\mathrm{D}$ is as snown in Fig. 24. Also, in the figure the plan view and the elevation of the polyhedric configuration have been given together with the global Cartesian coordinate system. The construct

$$
\operatorname{POL}(1,15,[0,0 ; 12,0])
$$

is a formex function representing a rule for transformation of a given formex $\mathrm{G}$ into a formex $\mathrm{D}$. The parameters $1,15,[0,0 ; 12,0]$, are parts of the rule defining the particulars of the transformation and are referred to as canonic parameters. The above function is referred to as a "polyhedron function". The polyhedron function can be used to create single layer or multi layer polyhedric configurations. The general form of the polyhedron function for single layer polyhedric configurations may be written as

$$
\operatorname{POL}(\mathrm{P}, \mathrm{R},[\mathrm{A} 1, \mathrm{~A} 2 ; \mathrm{B} 1, \mathrm{~B} 2]<<,\{\mathrm{F} 1, \mathrm{~F} 2, \ldots, \mathrm{Fn}\}>>\text { ) }
$$

where the first canonic parameter $\mathrm{P}$ is referred to as the "polyhedron code". The polyhedron code specifies the type of polyhedron which is to be used as the basis for the operation. Table 2 lists the code numbers for the Platonic and Archimedean polyhedra. The integer 1 given as the polyhedron code in the above polyhedron function specifies a tetrahedron. The "radius specifier" determines the size of the polyhedron by specifying the radius of the circumsphere, that is, the sphere that contains all the vertices of the polyhedron. This parameter is given as 15 units of length. The "locator" specifies the manner in which a given configuration is to be mapped onto a face of the polyhedron. To elaborate, consider the configuration shown in Fig. 23. Two corners of the configuration are denoted by the letters A and B. The configuration is intended to be placed on a face of the tetrahedron in such a way that $\mathrm{AB}$ fits an edge of the tetrahedron. This convention in conveyed by including the U1-U2 coordinates of A and $\mathrm{B}$ in the locator.

The last canonic parameter is referred to as the "face list". The role of the face list is to specify those faces of the polyhedron onto which the configuration is to be mapped. The face list is enclosed in special brackets which are referred to as option brackets. Absence of the face list in the polyhedron function implies that the configuration is to be mapped onto all the faces of that polyhedron. However, one has the option of generating only a part of the polyhedric configuration by specifying the required face numbers through the face list. The face numbers for the Platonic and Archimedean polyhedra are given in Figs. 1-5 and Figs. 8-20, respectively.

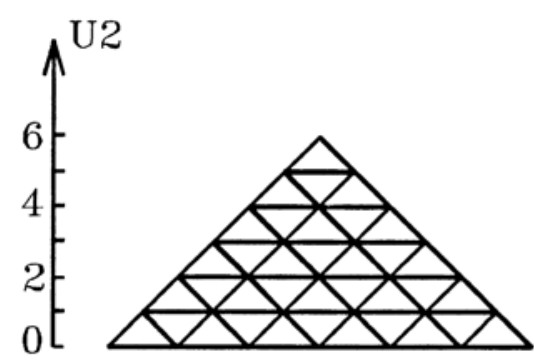

Fig. 21 Formex E.

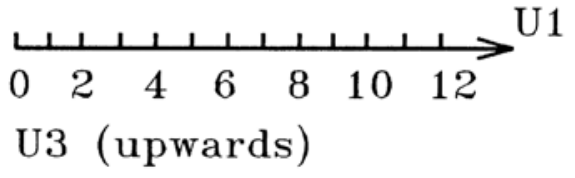

$\mathrm{D}=\mathrm{PEX}|\operatorname{POL}(1,15,[0,0 ; 12,0])| \mathrm{G}$

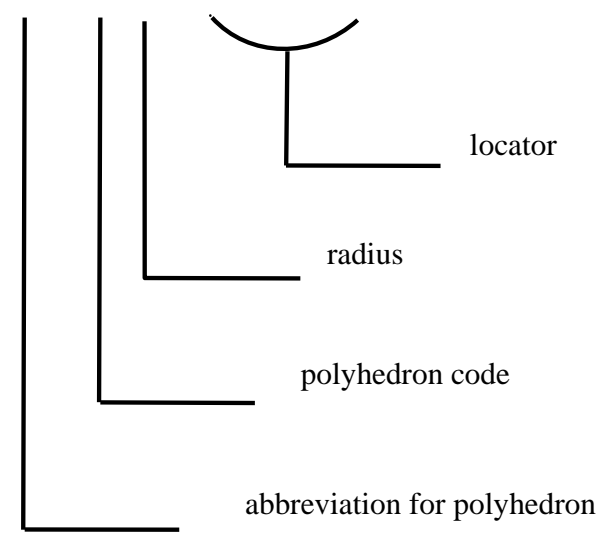

Fig. 22 Formian statement of polyhedron function. 


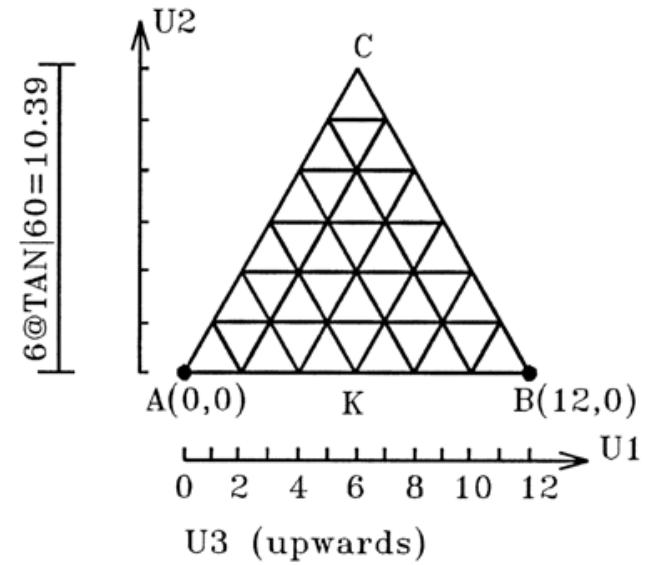

Fig. 23 Formex G.
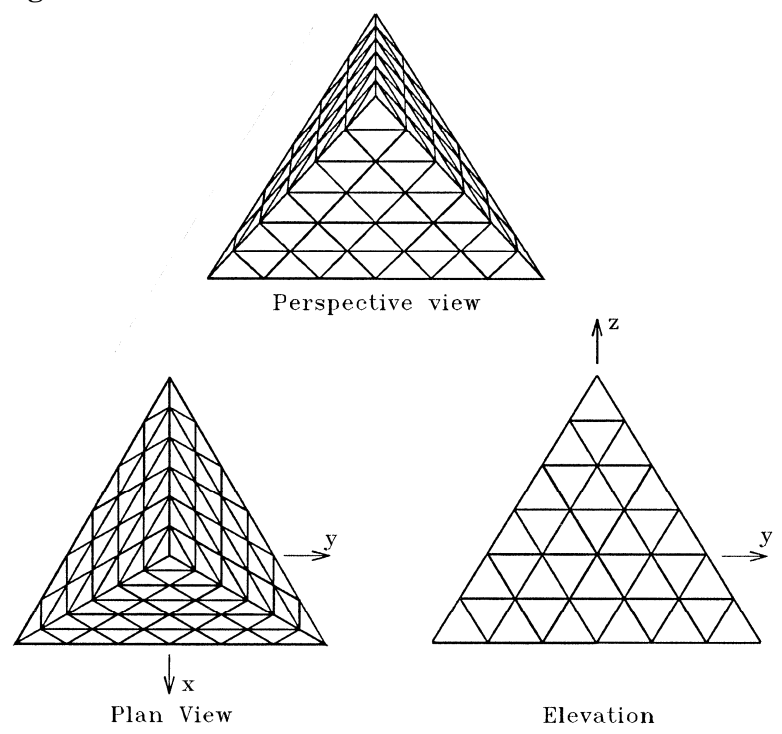

Elevation

Fig. 24 Graphical representation of formex variable $D$.

\subsection{Polyhedron Code}

The polyhedron code $\mathrm{P}$ is a nonzero integer defining the type of polyhedron. The value of the polyhedron code P may be from 1 to 18 as shown in Table 2 . In this table the right handed versions of the snub cube and snub dodecahedron have been assigned positive values whereas the left handed versions have been assigned negative values.

\subsection{Symmetry Operations}

This section is devoted to the description of the process which is used for the creation of the tetrahedral configuration that described above in section 3. This process is based on the constructs of formex functions [8]. To begin with consider the configuration shown in
Fig. 23. The polyhedron function for the creation of the tetrahedral configuration of edge length $L=24.5$ may be written as

$$
\mathrm{D}=\mathrm{PEX}|\operatorname{POL}(1,15,[0,0 ; 12,0])| \mathrm{G}
$$

where $\mathrm{G}$ is a formex variable representing the compret of the configuration of Fig. 23 and where formex variable $\mathrm{D}$ represents the entire tetrahedral configuration of Fig. 24. At this stage the U1-U2-U3 axes are assumed, to coincide with the $x-y-z$ axes of the global Cartesian coordinate system.

Finally, it should also be mentioned that in the formulation of formex variable $\mathrm{D}$ the PEX function has been used to remove all the doubly represented elements along the edges of the tetrahedral configuration (Fig. 25).

Procedures similar to the ones which are described above are followed for the creation of all the Platonic and Archimedean polyhedral. Each one of the procedures consists of a series of Formian statements. Each of the Formian statements involves a number of formex functions [9]. These formex functions are used to imply dilatation, translation, rotation or reflection of a given configuration. The values of the canonic parameters which are involved in the formex function are specified in relation to the $x-y-z$ global Cartesian coordinate system, which is given in each one of the Figs. 1-5 and 8-20. The procedure which has been followed for the creation of the three non $x-y$ faces of the tetrahedral configuration is referred to as "Procedure 2.1" and its general form is given in the sequel. Also, the procedure which has been followed for the creation of the bottom face of the tetrahedral configuration is referred to as "Procedure 4.1" and its general form is also given in the sequel. However, for the creation of the Platonic polyhedra some other
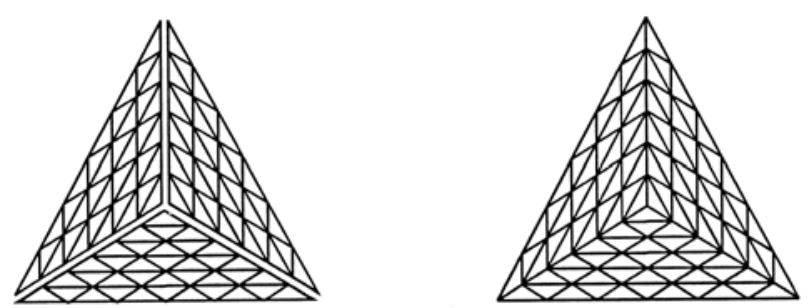

Fig. 25 Graphical demonstration of PEX function. 
procedures are needed and their general description is also given in Fig. 26.

\section{Basic Procedures for the Creation of the Platonic Polyhedra}

For the creation of the Platonic polyhedra some basic procedures have been followed throughout this paper and depend on the type of face to be created, that is top $\mathrm{x}-\mathrm{y}$ face, non $\mathrm{x}-\mathrm{y}$ face and bottom $\mathrm{x}-\mathrm{y}$ face. Seven different procedures are followed in the creation of the three types of faces of the polyhedric configurations that are based on the Platonic polyhedra. Procedure 1 deals with the creation of the top $x-y$ face of the polyhedric configuration. Procedures 2.1 and 2.2 deal with the creation of the non $x-y$ faces of the top part of the polyhedric configuration. Procedures 3.1 and 3.2 deal with the creation of the non $x-y$ faces of the bottom part of the polyhedric configuration and Procedures 4.1 and 4.2 deals with the creation of the bottom $x-y$ face of the polyhedric configuration.

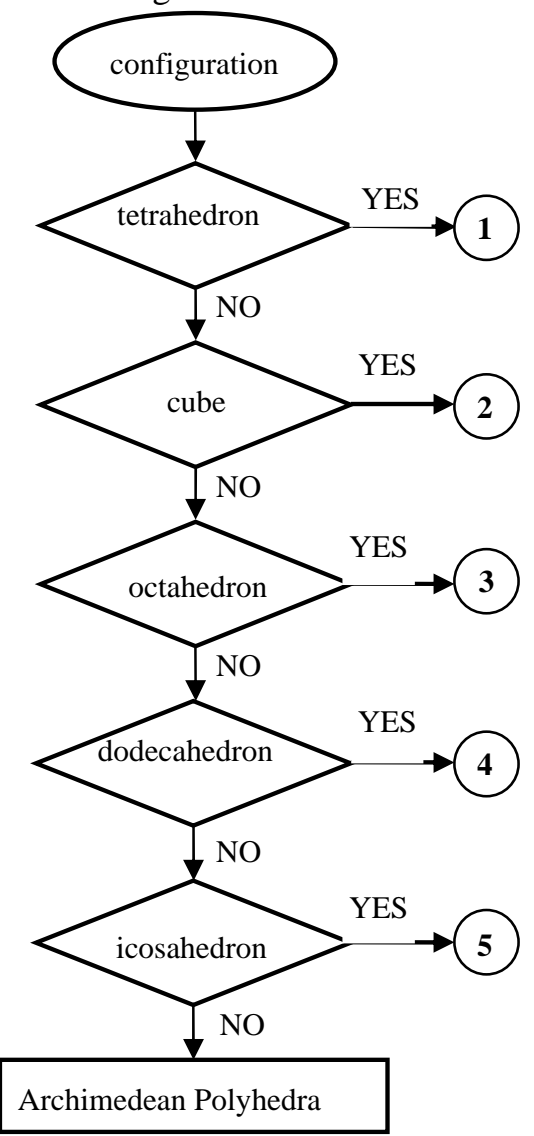

Fig. 26 Flow chart for the creation of the Platonic.

\subsection{Top x-y Face}

The top $x-y$ face is the face which is parallel to the $x-y$ plane and has the maximum $z$ coordinate. Of the Platonic polyhedra only the cube and the dodecahedron have a top x-y face (Fig. 2 and Fig. 4). Procedure 1 is adopted for the creation top $\mathrm{x}-\mathrm{y}$ face of a polyhedric configuration.

4.1.1 Procedure 1

$\mathrm{P}=\operatorname{VERAD}(0,0)|\operatorname{DILID}(\mathrm{L}, \mathrm{L})| \mathrm{TRANID}(-\mathrm{Tx} 1, \mathrm{Ty}) \mid \mathrm{G}$ $\mathrm{Q}=$ TRANIS(Tx2, Tz) $\mid \mathrm{P}$

where the terms which are used in the above formulations are as follows:

G: formex variable representing the compret of a given configuration

L: edge length of the polyhedric configuration

Tx1, Tx2: amount of translation along the $\mathrm{x}$ axis

Ty: amount of translation along the $y$ axis

Tz: amount of translation along the $\mathrm{z}$ axis

\subsection{Non x-y Faces, Top Part}

A non $x-y$ face is a face which is not parallel to the $\mathrm{x}-\mathrm{y}$ plane. The top part of a polyhedric configuration is the part which is above the $\mathrm{x}-\mathrm{y}$ plane. All the Platonic polyhedra have non $\mathrm{x}-\mathrm{y}$ faces (Figs. 1-5). Procedures 2.1 and 2.2 are used for the creation of the non $x-y$ faces of the top part of a Platonic polyhedron. Let the line $A B$ represents an edge of the configuration that is to be mapped on a non $x-y$ face of the top part. Procedure 2.1 is used for the creation of a non $x-y$ face when the line AB is parallel to the $\mathrm{y}$ axis and lies on a plane parallel to the $\mathrm{x}-\mathrm{y}$ plane. Procedure 2.2 is used for the creation of a non $x-y$ face when the configuration which is mapped on to a face of the polyhedric form is to be reflected.

4.2.1 Procedure 2.1

$\mathrm{P}=\mathrm{VERAD}(0,0)|\mathrm{DILID}(\mathrm{L}, \mathrm{L})| \mathrm{TRANID}(-\mathrm{Tx} 1,-\mathrm{Ty}) \mid \mathrm{G}$

$\mathrm{Q}=\mathrm{TRAN}(1, \mathrm{Tx} 2)|\mathrm{VERAS}(0,0,-\mathrm{Ry})| \mathrm{P}$

$\mathrm{S}=\mathrm{ROSAD}(0,0, \mathrm{~F}, \mathrm{Rz})|\mathrm{TRAN}(3,-\mathrm{Tz})| \mathrm{Q}$

where the terms G, L, Tx1, Tx2, Ty and Tz which are used in the above formulations are as given above. $\mathrm{F}$ refers to number of faces to be created. 
4.2.2 Procedure 2.2

$\mathrm{P}=\mathrm{VERAD}(0,0)|\mathrm{DILID}(\mathrm{L}, \mathrm{L})| \mathrm{TRANID}$ (-Tx1, -Ty)|

$\mathrm{Q}=\operatorname{REF}(3,0)|\operatorname{VERAS}(0,0,-\mathrm{Ry})| \mathrm{P}$

$\mathrm{S}=\mathrm{ROSAD}(0,0, \mathrm{~F}, \mathrm{Rz})|\mathrm{TRANIS}(\mathrm{Tx} 2,-\mathrm{Tz})| \mathrm{Q}$

The terms which are given in the above formulations should be interpreted in a way similar to the ones given in Procedure 2.1. Procedure 2.2 is similar to Procedure 2.1 except that in addition to the other stages it involves a reflection with respect to the $\mathrm{x}-\mathrm{y}$ plane.

\subsection{Non x-y Faces, Bottom Part}

\subsubsection{Procedure 3.1}

Some of the Platonic polyhedra have the same arrangement of the non $x-y$ faces in the top part as in the bottom part. In this case, the entire top part of the corresponding polyhedric configuration is reflected with respect to the $\mathrm{x}-\mathrm{y}$ plane to obtain the bottom part of that polyhedric configuration. A Formian statement describing this operation may be given as

$$
\mathrm{BTM}=\operatorname{REF}(3,0) \mid \mathrm{TOP}
$$

where formex variable TOP represents the top part of the polyhedric configuration and the formex variable BTM represents the bottom part.

\subsubsection{Procedure 3.2}

Two stages are identified in this procedure. The first stage is the same as Procedure 3.1. In the second stage, the reflection of the top part of the polyhedric configuration is followed by an anticlockwise rotation around the $\mathrm{z}$ axis by an appropriate angle to obtain the bottom part of the polyhedric configuration. A Formian statement describing the Procedure 3.2 may be given

$\mathrm{BTM}=\operatorname{VERAD}(0,0, \mathrm{Rz})|\operatorname{REF}(3,0)| \mathrm{TOP}$

where the canonic parameter $\mathrm{Rz}$ defines the angle of rotation about the $\mathrm{z}$ axis.

\subsection{Bottom x-y Face}

The bottom $x-y$ face is the face which is parallel to the $\mathrm{x}-\mathrm{y}$ plane and has the minimum $\mathrm{z}$ coordinate. All the Platonic polyhedra except the octahedron and the icosahedron have a bottom x-y face (Figs. 1, 2 and 4). Procedures 4.1 and 4.2 are used for the creation of the bottom x-y face of a Platonic polyhedric configuration. Let the line $\mathrm{AB}$ represents an edge of a polyhedric configuration. Procedure 4.1 is used for the creation of the bottom $x-y$ face when the line AB is parallel to the $y$ axis and the Procedure 4.2 is used when line $A B$ is not parallel to the y axis.

4.4.1 Procedure 4.1

$\mathrm{P}=\mathrm{VERAD}(0,0)|\mathrm{DILID}(\mathrm{L}, \mathrm{L})| \mathrm{TRANID}(-\mathrm{Tx} 1,-\mathrm{Ty}) \mid \mathrm{G}$

$\mathrm{Q}=\operatorname{REF}(3,0)|\operatorname{VERAS}(0,0,-90)| \mathrm{P}$

$\mathrm{S}=$ TRANIS(Tx2,-Tz)|VERAS(0, 0, -90) |Q

where the terms G, L, Tx1, Tx2, Ty and Tz which are used in the above formulations are as given above. $\mathrm{Rz}$ refers to angle of rotation about the $\mathrm{z}$ axis in degrees.

4.4.2 Procedure 4.2

$\mathrm{P}=\mathrm{VERAD}(0,0)|\mathrm{DILID}(\mathrm{L}, \mathrm{L})|$ TRANID(-Tx1, -Ty)|G

$\mathrm{Q}=\mathrm{REF}(3,0)|\operatorname{VERAS}(0,0,-90)| \mathrm{P}$

$\mathrm{S}=\mathrm{TRANIS}(\mathrm{Tx} 2,-\mathrm{Tz})|\operatorname{VERAS}(0,0,-90)| \mathrm{Q}$

$\mathrm{W}=\operatorname{VERAD}(0,0, \mathrm{Rz}) \mid \mathrm{S}$

The terms which are given in the above formulations should be interpreted in a way similar to the ones given in Procedure 4.1. Procedure 4.2 is similar to Procedure 4.1 except that in addition to the other stages it involves a rotation around the $\mathrm{z}$ axis and is only applied when the bottom $\mathrm{x}-\mathrm{y}$ pentagonal face of a dodecahedral configuration is to be created. Table 3 summarises the process for the creation of each one of the Platonic polyhedra. The first column of Table 3 lists the Platonic polyhedra. The second column corresponds to Procedure 1 and deals with the creation of the top $x-y$ face of a polyhedric configuration. Columns 3 to 6 deal with the creation of the non $x-y$ faces of a polyhedric configuration. In particular, columns 3 and 4 deal with the non $x-y$ faces that constitute the top part of a polyhedric configuration and correspond to Procedures 2.1 and 2.2, respectively. Columns 5 and 6 deal with the creation of the non $x-y$ faces that, constitute the bottom part of the polyhedric configuration and correspond to Procedures 3.1 and 3.2. Finally, columns 7 and 8 deal with the creation of the bottom $x-y$ faces of a polyhedric configuration. The number and the type of faces that are created in each of the procedures are 
given at the top and bottom of a cross respectively. Furthermore, Table 3 may be interpreted as follows: Let it be required to generate a polyhedric configuration based on a tetrahedron. For the creation of the three non $\mathrm{x}-\mathrm{y}$ triangular faces Procedure 2.1 is applied and for the creation of the bottom $\mathrm{x}-\mathrm{y}$ triangular faces Procedure 4.1 is applied.

As the next example, suppose that a polyhedric configuration based on a dodecahedron is to be generated. The dodecahedron has a top $x-y$ face, a bottom $x-y$ face and ten non $x-y$ faces where five of them constitute the top part and the other five constitute the bottom part of the dodecahedral configuration. For the creation of the top $x-y$ pentagonal face Procedure 1 is applied and for the creation of the five non $\mathrm{x}$-y pentagonal faces that constitute the top part of the dodecahedral configuration procedure 2.2 is applied. Furthermore, Procedures 3.2 and 4.2 are applied for the creation of the five non $x-y$ pentagonal faces of the bottom part and the bottom $x-y$ face of the dodecahedral configuration, respectively.

To further clarify the approach for the creation of each of the Platonic polyhedra a flow chart has been given for each one of them. The main body of the flow chart consists of five rhombic boxes, where each box represents a Platonic polyhedron (Fig. 26). The numbers that are given on the side of each box correspond to the ones in Table 2. Smaller flow charts with rectangular boxes have been given for each polyhedron describing the order of the procedures that have to be followed for the creation of a polyhedric configuration (Figs. 27-31). For instance, suppose that is required to generate an icosahedron. It may be seen from Fig. 5 that an icosahedron has twenty triangular non $x-y$ faces and represents the same arrangement of faces in the top part as in the bottom part. For the creation of the top part of the icosahedral configuration Procedures 2.1 and 2.2 are applied. In particular, Procedure 2.1 is applied for the creation of the five triangular non $x-y$ faces of the top part (5 T Faces) and Procedure 2.2 is applied for the creation of the other five non $\mathrm{x}-\mathrm{y}$ reflected triangular faces (5 $\mathrm{T}$ Faces). Finally, Procedure 3.2 is applied for the creation of the ten triangular faces (10 $\mathrm{T}$ Faces) that constitute the bottom part of the icosahedral configuration. The letter $\mathrm{T}, \mathrm{S}$, or $\mathrm{P}$ which are given at the bottom of the rectangular boxes in the smaller flow charts represent a triangular, a square or a pentagonal polyhedral face respectively.

As the next example, consider the configuration shown in Fig. 32. This is a view of a polyhedric configuration obtained by mapping the configuration of Fig. 33 onto three faces of a cube. The formulation

Table 3 The process for the creation of each one of the Platonic polyhedral.

\begin{tabular}{|c|c|c|c|c|c|c|c|}
\hline \multirow{3}{*}{ Polyhedron } & \multirow{3}{*}{$\begin{array}{c}\text { Procedure } 1 \\
\text { Top face }\end{array}$} & \multicolumn{4}{|c|}{ Non $x$-y faces } & \multirow{2}{*}{\multicolumn{2}{|c|}{ Bottom face }} \\
\hline & & \multicolumn{2}{|c|}{ Top part } & \multicolumn{2}{|c|}{ Bottom part } & & \\
\hline & & Procedure 2.1 & Procedure 2.2 & Procedure 3.1 & Procedure 3.2 & Procedure 4.1 & Procedure 4.2 \\
\hline Tetrahedron & & & & & & & \\
\hline Cube & & & & & & & \\
\hline Octahedron & & & & & & & \\
\hline Dodecahedrc & & & & & & & \\
\hline Icosahedron & & & & & & & \\
\hline
\end{tabular}




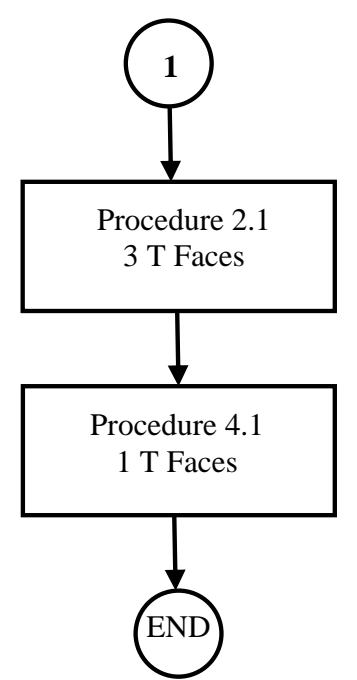

Fig. 27 Flow chart for the creation of a tetrahedron.

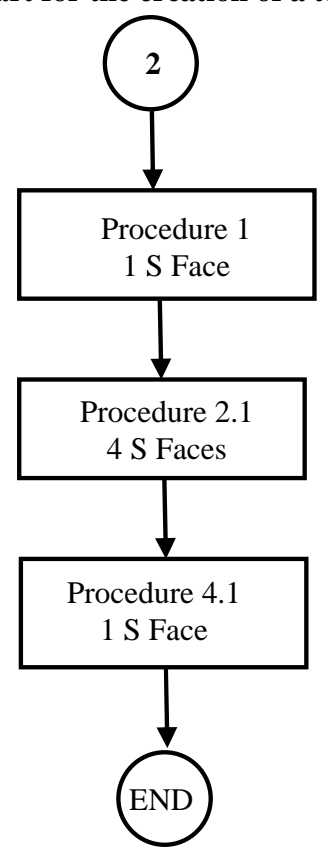

Fig. 28 Flow chart for the creation of a cube.

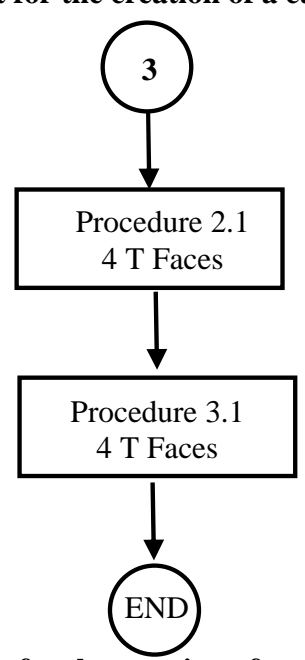

Fig. 29 Flow chart for the creation of an octahedron.

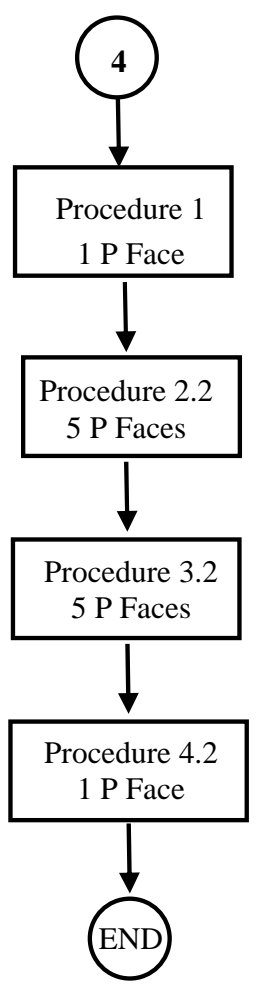

Fig. 30 Flow chart for the creation of a dodecahedron.

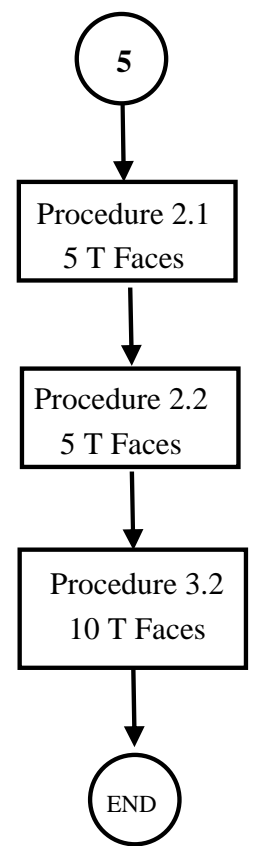

Fig. 31 Flow chart for the creation of an icosahedron.

of formex variable $\mathrm{C} 1$ that represents the polyhedric form of Fig. 32 may be given as

$$
\mathrm{C} 1=\mathrm{POL}(2,10,[0,0 ; 12,0],[1 ; 3]) \mid \mathrm{P} 1
$$

where the argument $\mathrm{P} 1$ represents the configuration of Fig. 33 and may be given as

$$
\text { P1=RINID(6,6,2,2) | ROSAD(1,1) | [0,0; 1,1] }
$$




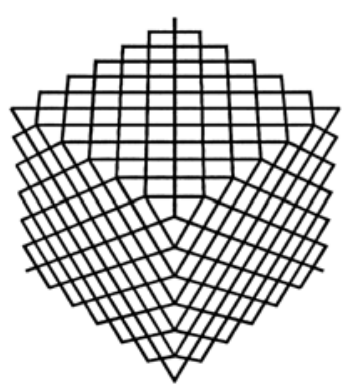

Fig. 32 A polyhedric form.

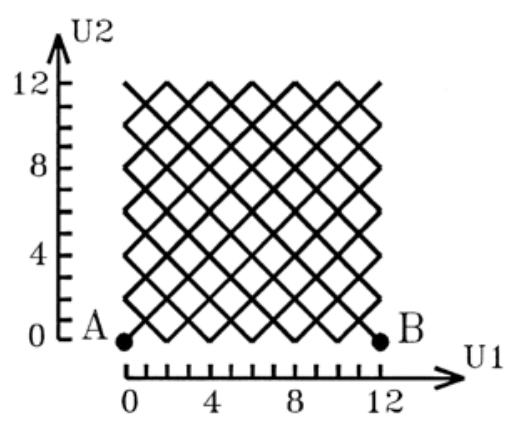

Fig. 33 Form of P1.

The new points to be noticed in this example are:

(1) The type of polyhedron may be changed. A cube has been used as the base polyhedron in Fig. 32 instead of the tetrahedron of Fig. 24.

(2) The part of the polyhedron on which the configuration mapped may be varied. The first, second and third face of the cube have been used to obtain the polyhedric form of Fig. 32 whereas the entire tetrahedron has been create in Fig. 24. Therefore, the face list has been used to specify the faces 1 to 3 of the cube onto which the configuration is to be mapped. The face numbers of the Platonic polyhedra are given in Figs. 1-5.

In creating a polyhedric form, it is possible to use different patterns for different faces of the base polyhedron. For instance, in the case of the polyhedric configuration of Fig. 34, the diagonal pattern of Fig. 33 is placed onto the second and third faces of the cube and the pattern of Fig. 35 is placed onto the first face. The formulation of formex variable C2 that represents the polyhedric form of Fig. 34 is given as C2=POL(2,10,[0,0; 12,0], [2; 3])|P1\#POL(2,10,[0,0; 12,0],1) | P2 where the argument P2 represents the configuration of Fig. 35 and may be given as

$$
\mathrm{P} 2=\mathrm{RINID}(6,6,2,2) \mid[0,0 ; 2,0 ; 2,2 ; 0,2]
$$

An important point to be noticed here is that the pattern of Fig. 35 consists of "tile" elements whereas the pattern of Fig. 33 consists of "line" elements. The difference between the element types is implied through the formex formulations. Namely, in the formulation of P1 an element is specified by the coordinates of its two end points, whereas in the formulation of $\mathrm{P} 2$ an element is specified by the coordinates of its four corner points. In general, there are no restrictions regarding the types of elements that may be involved in configurations for creation of polyhedric forms.

A configuration that is mapped onto a face of a polyhedron need not necessarily "fill" the face or "match" the boundaries of the face, as demonstrated by the polyhedric configuration shown in Fig. 36. Here, the configuration of Fig. 37 which has a rectangular shape of boundary is mapped onto the first face of the cube and the configuration of Fig. 38 which has a triangular shape of boundary is mapped onto the second and third faces of the cube. The configuration that is placed onto the first face extends beyond the edges of the face and the configuration that is placed onto the second and the third faces cover the face only partially. The formex formulation of formex variable C1 that represents the polyhedric form of Fig. 36 may be given as

C1=POL(2,10, [0,0; 12,0], 1)|P3\#POL(2,10, [0,0; 12,0], [2; 3])|P4

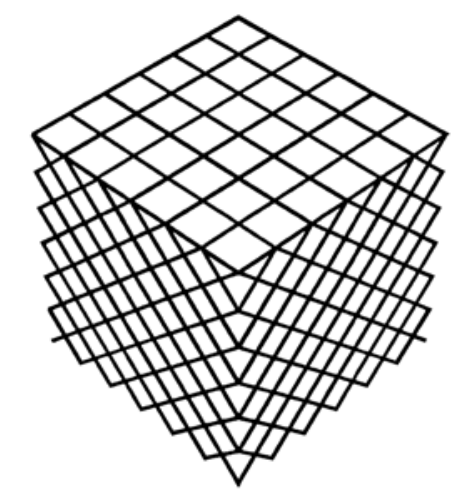

Fig. 34 A polyhedric form. 


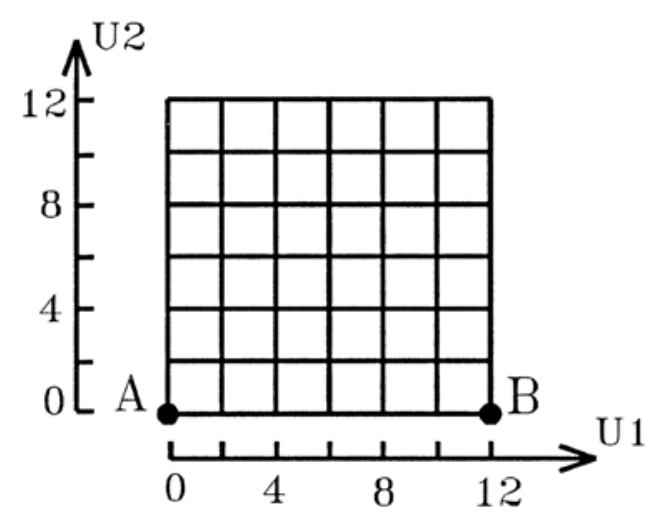

Fig. 35 Form of P2.

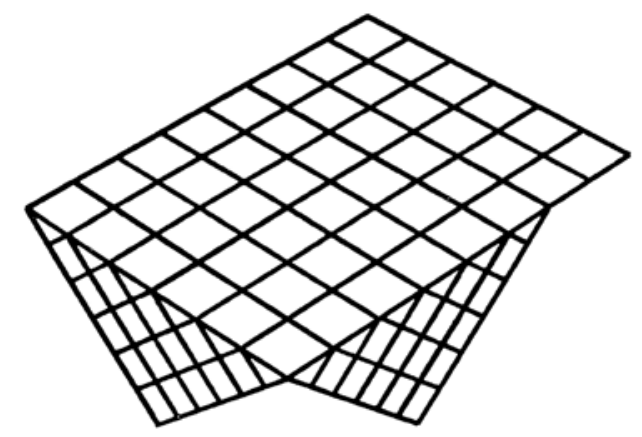

Fig. 36 A polyhedric form.

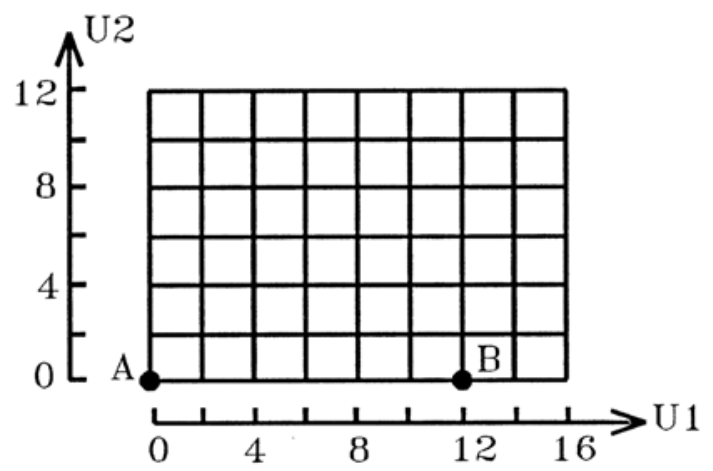

Fig. 37 Form of P3.

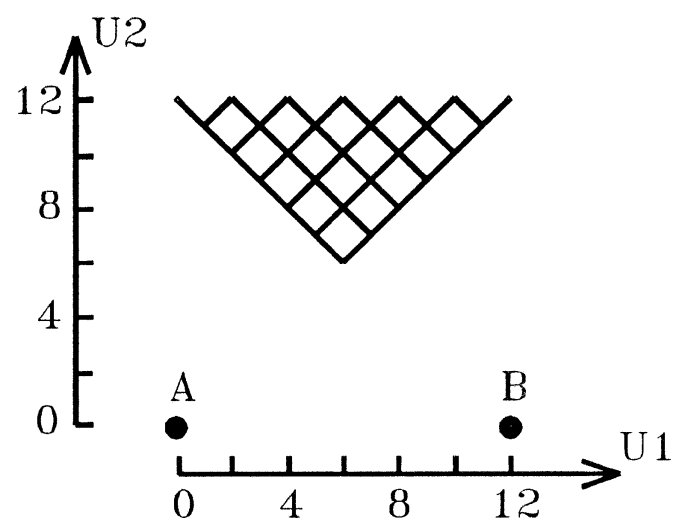

Fig. 38 Configuration of formex variable $\mathbf{P 4}$. where the argument P3 represents the configuration of Fig. 37 and may be given as

$$
\text { P3=RINID }(8,6,2,2) \mid[0,0 ; 2,0 ; 2,2 ; 0,2]
$$

and the argument $\mathrm{P} 4$ represents the configuration of Fig. 38 and may be given as

$$
\mathrm{P} 4=\mathrm{JUN}([1,2,1],[-2,13 ; 6,5 ; 14,13]) \mid \mathrm{P} 1
$$

So far, the polyhedron function has been used to generate single layer polyhedric configurations. However, the polyhedron function can be used to generate multi-layer polyhedric configurations. For example, consider the configuration shown in Fig. 39. This is a view of a double layer polyhedric configuration which is based on the top five faces of two concentric icosahedra. The double layer configuration used for mapping is shown in Fig. 40 together with the normat U1-U2-U3 for the formex formulation. A formex formulation for this double layer triangular configuration may be given as T=GENID(5,5,2,SQRT|3,1,-1)|ROSAD(1,SQRT|3/3,3, 120) $\mid\{[0,0,2 ; 2,0,2],[0,0,2 ; 1, \mathrm{SQRT} \mid 3 / 3,1]\}$ $\mathrm{B}=\mathrm{GENID}(4,4,2, \mathrm{SQRT} \mid 3,1,-1) \mid \operatorname{ROSAD}(2,2, \mathrm{SQRT} \mid 3 / 3$ ,3, 120) | [1, SQRT|3/3,1; 1,SQRT|3/3,1]

In Figs. 39-40 the top layer elements are drawn in thick lines and the bottom layer elements as well as the web elements are drawn in thin lines. The formulation of formex variable $\mathrm{C} 4$ that represents the polyhedric form of Fig. 39 may be given as C4=PEX|POL(5, [10; 12], \{[1, SQRT|3/3; 9, SQRT| $3 / 3],[0,0 ; 10,0]\},[1 ; 5]) \mid(\mathrm{T} \# \mathrm{~B})$

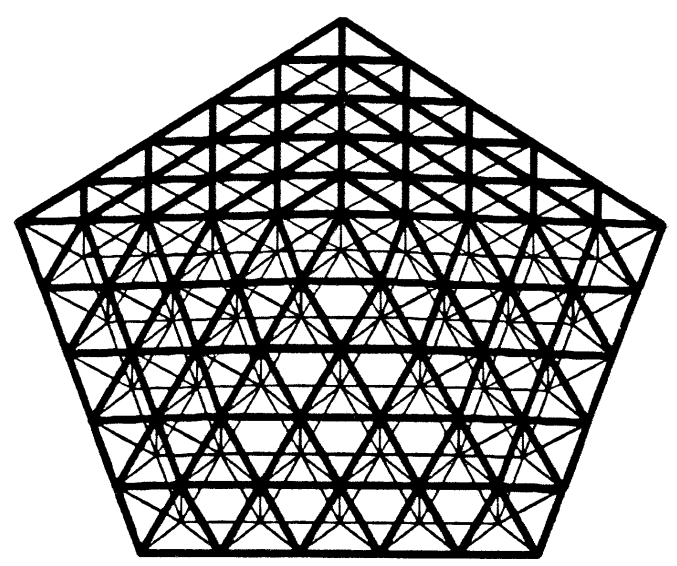

Fig. 39 A graphical representation of a double layer polyhedric configuration. 


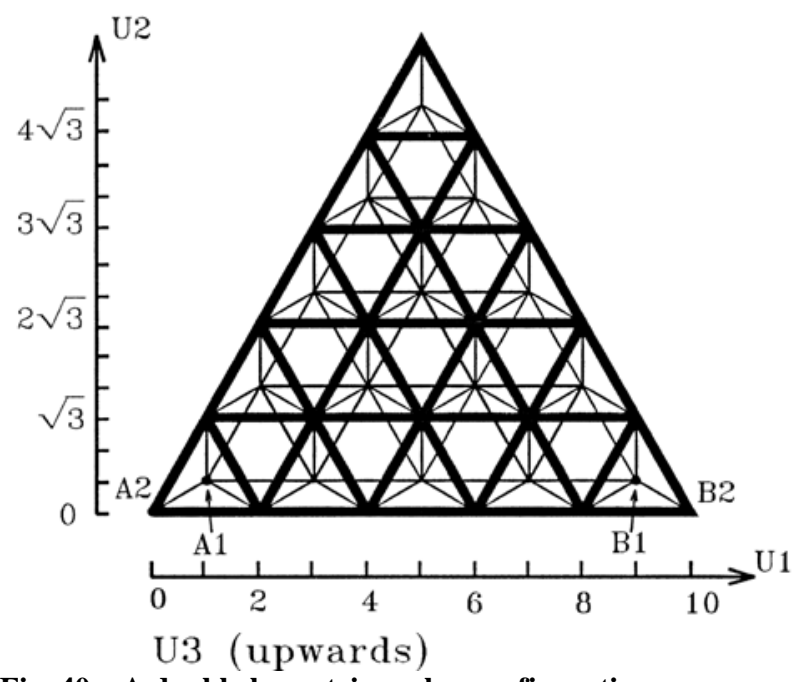

Fig. 40 A double layer triangular configuration.

The new points to be noticed in this example are:

(1) In formulating the configuration of Fig. 40 each layer has an identification number. The identification number of the bottom layer is 1 and that of the top layer is 2. These identification numbers appear as "third direction coordinates" in the formex formulations.

(2) The radius specifier in formex variable C4 contains two entries. The first entry, that is, 10 gives the radius of the circumsphere of the base polyhedron for the first layer (bottom layer) and the second entry, that is, 12 gives the radius of the circumsphere of the base polyhedron for the second layer (top layer).

(3) The locator in C4 contains two parts. The first part of the locator specifies the coordinates of points A1 and B1 in Fig. 40 that should fit the end vertices of an edge of the base polyhedron of the first layer (bottom layer). The second part of the locator specifies the coordinates of points A2 and B2 in Fig. 40 that should fit the end vertices of an edge of the base polyhedron of the second layer (top layer). Situations when polyhedric configurations involve more than two layers are dealt with in an analogous manner. In such case, the general form of the polyhedron function may be written as

POL(T,[R1;R2;...Rn],\{[A1;B1],[A2;B2],..,[AN;BN] \}$<<\{, \mathrm{F} 1, \mathrm{~F} 2, \ldots, \mathrm{Fn}\}>>)$

where the construct $[\mathrm{R} 1 ; \mathrm{R} 2 ; \ldots ; \mathrm{Rn}]$ lists the radii of the circumspheres of the base polyhedra for the $\mathrm{n}$ layers.
The locator contains $n$ cantles where each one of the cantles specifies the coordinates of points A and B that should fit the end vertices of an edge of the base polyhedron for each one of the $n$ layers.

So far, polyhedric configurations based on the Platonic polyhedra have been generated. However, the polyhedron function can be used to create polyhedric configurations based on the Archimedean polyhedra. In the case of Archimedean polyhedra the faces are not all of the shame shape. In spite of this, one may create a polyhedric form by mapping a single configuration on all or some of the faces of an Archimedean polyhedron. However, in creating polyhedric forms that are based on Archimedean polyhedra, normally one would use different configurations for different face shapes. For instance, the polyhderic configuration of Fig. 41 is based on a cuboctahedron and is obtained by mapping the configuration of Figs. 42 and 43 onto the triangular and square faces, respectively.

A Formian statement describing this operation may be given as

$\mathrm{C} 5=\operatorname{PEX} \mid(\operatorname{POL}(7,10,[0,0 ; 12,0],[1 ; 8] \mid \mathrm{P} 5 \quad \# \ldots$ $\operatorname{POL}(7,10,[0,0 ; 12,0],[9 ; 14]) \mid \mathrm{P} 6)$

where $\mathrm{P} 5$ is a formex variable representing the compret of the configuration of Fig. 42 and may be given as P5=GENID(6,6,2,SQRT|3,1,-1)|ROSAD(1,SQRT|3/3, $3,120)[[0,0 ; 2,0]$

and $\mathrm{P} 6$ is a formex variable representing the compret of the configuration of Fig. 43 and may be given as P6 = LUX([6,6]) |ROSAD(6,6) | BB(1,1/SQRT|3) | P5

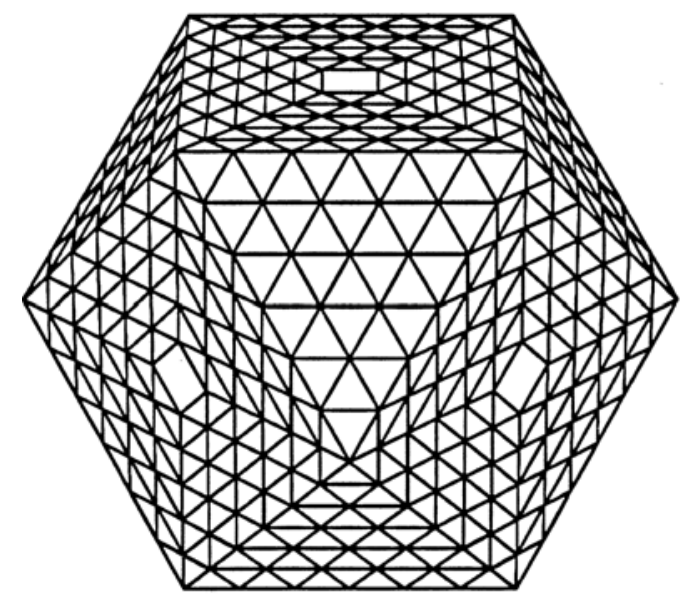

Fig. 41 A graphical representation of the cuboctahedron. 


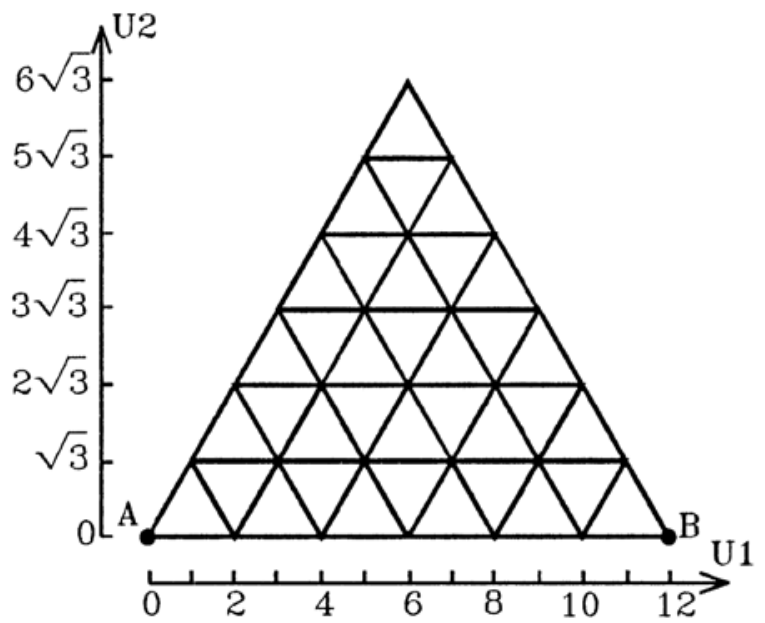

Fig. 42 A triangular form.

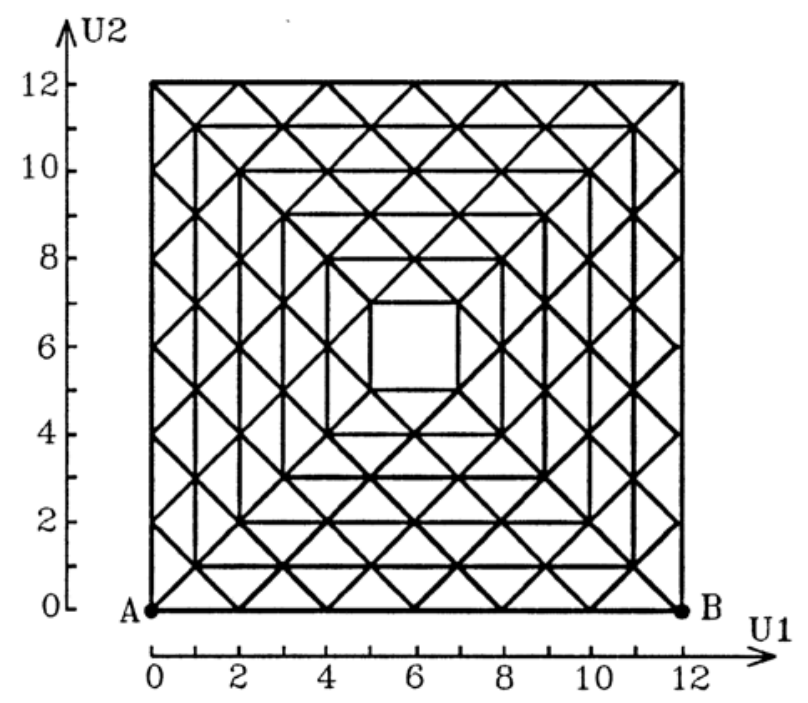

Fig. 43 A square form.

The process which has been followed for the creation of the cuboctahedral configuration is similar to the one followed for the creation of the tetrahedral configuration in section 3.2. The cuboctahedron has a top $\mathrm{x}-\mathrm{y}$ square face, eight triangular and four square non $\mathrm{x}-\mathrm{y}$ faces and a bottom x-y square face Fig. 9. Procedures similar to the ones described above are used for the creation of all the Archimedean polyhedra.

\section{Geodesic Forms}

Geodesic forms allow effective use of material and space and may be employed to create architecturally interesting and economic building structures. They are presently used in a number of specialized areas of construction such as domes for arenas, cultural centres, exhibition halls and Olympic facilities [10]. Their widespread use has been obstructed by the difficulty in defining their geometry [11]. This problem has presented a challenge for engineers and architects for decades. Many attempts have been made throughout the world to evolve techniques that deal with the data generation of geodesic forms. However, the approach presented in this paper provides a basis for the configuration processing of geodesic forms in a compact and readily understood manner.

Most of the existing geodesic domes have been obtained from the radial projection of the triangular faces of an icosahedron on a sphere. However, in this paper a geodesic dome may be obtained by projecting a polyhedric configuration on a surface. For the projection the tractation retronorm will be applied.

\section{An Example}

Consider the configuration shown in Fig. 44. This is obtained by projecting the polyhedric configuration of Fig. 45 on a sphere which is concentric with the icosahedron using the centre of the sphere as the centre of projection. A Formian statement describing this operation may be given as shown in Fig. 46 where the formex variable $\mathrm{P}$ represents the top five faces of the icosahedral configuration of Fig. 45 and may be given as

$$
\mathrm{P}=\operatorname{PEX}|\operatorname{POL}(5,10,[0,0 ; 18,0],[1 ; 5])| \mathrm{E}
$$

Formex variable E represents the compret of Fig. 47 and may be given as

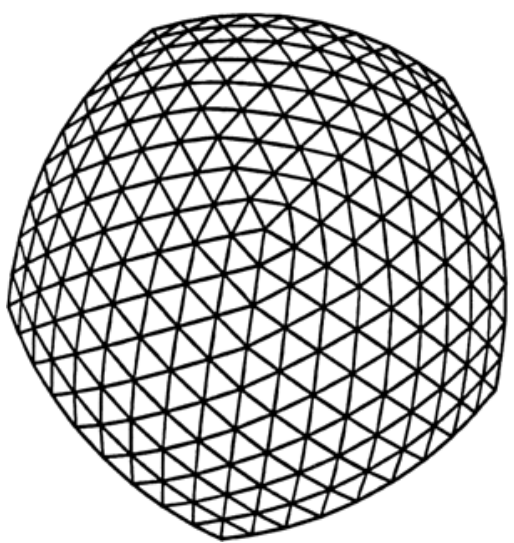

Fig. 44 Projection on a sphere. 


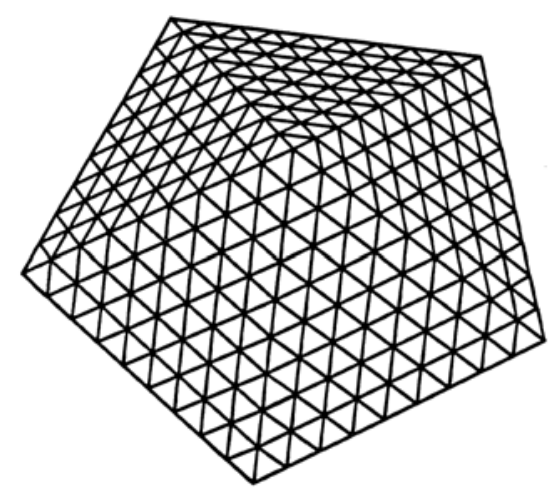

Fig. 45 A polyhedric form.

$\mathrm{D}=\operatorname{TRAC}(4,1,0,0,0,10,13) \mid \mathrm{P}$

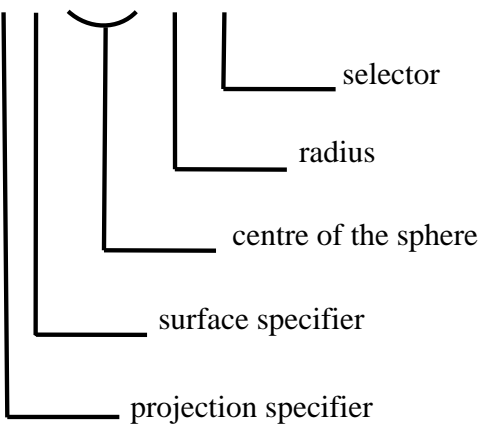

abbreviation for tractation specifier

Fig. 46 Formian statement of tractation retronorm.

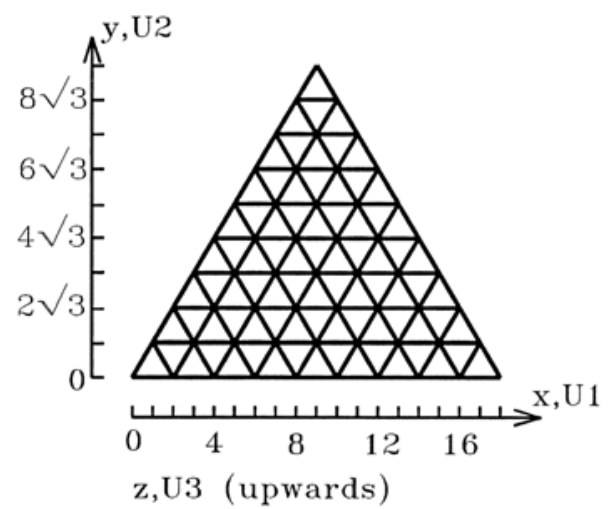

Fig. 47 A triangular configuration.

\section{E=GENID $(9,9,2$, SQRT $\mid 3,1,-1) \mid\{[0,0 ; 2,0],[2,0 ; 1$, SQRT \\ |3],[1,SQRT|3;0,0]\}}

The configuration of Fig. 44 is an example of a "geodesic form” or "geodesic configuration".

The constituent parts of formex variable D are as follows:

TRAC is an abbreviation for tractation and is followed by a sequence of parameters enclosed in parentheses. The construct that consists of TRAC together with the ensuing parameter list is referred to as a "tractation retronorm". The "projection specifier" specifies the type of projection to be used. The projection specifier may have the value 1,2,3 or 4 indicating central, parallel, axial or radial projection, respectively. The projection specifier in Fig. 46 is given as 4 , implying radial projection. The "surface specifier" specifies the type of surface on which projection is to be made. The surface specifier for formex variable $\mathrm{D}$ is given as 1 implying a sphere and this is followed by the coordinates of the centre of the sphere $(0,0,0)$ and the number 10 that specifies the radius of the sphere. The selector specifies the course of action to be taken when the projection of a point cannot be determined uniquely.

As the next example consider the configuration shown in Fig. 48. This is a view of a geodesic form obtained by projecting the icosidodecahedral configuration of Fig. 49 onto an ellipsoid using central projection. A Formian statement describing this operation may be given as

$$
\text { D1=TRAC(1,0,0,-10,2,0,0,-5,11,15,20,13)|P1 }
$$

where the formex variable $\mathrm{P} 1$ represents the polyhedric form of Fig. 49 and may be given as

P1=POL(12,10,[0,0;8,0],[1;10])|E1 \# POL(12,10,[0,0; 8,0], [21; 26]) | E2

Formex variable E1 represents the compret of the pattern on the triangular faces of the icosidodecahedral configuration Fig. 50.

Formex variable E2 represents the compret of the pattern on the pentagonal faces of the icosidodecahedral configuration Fig. 51.

A geodesic form may have more than one layer. For example, consider the configuration shown in Fig. 52. This is a view of a double layer geodesic form obtained by projecting the polyhedric configuration of Fig. 53 on two concentric spherical surfaces using radial projection. A Formian statement describing this operation may be given as

D3=TRAC $([4,1,4,1,0,0,0,10,13],[4,2,4,1,0,0,0,12,13]) \mid$ P2 
where the formex variable P2 represents the polyhedric form of Fig. 53 and may be given as

P2=PEX|POL(5,[10;12],\{[1,SQRT|3/3; 11,SQRT|3/3], $[0,0 ; 12,0]\},[1 ; 5]) \mid(\mathrm{T} \# \mathrm{~B})$

formex variable $\mathrm{T}$ and $\mathrm{B}$ represent the compret of the double layer configuration of Fig. 54 and may be given as

T=GENID(6,6,2,SQRT|3,1,-1)|ROSAD(1,SQRT|3/3,3 ,120) $\mid\{[0,0,2 ; 2,0,2],[0,0,2 ; 1, \mathrm{SQRT} \mid 3 / 3,1]\}$

$\mathrm{B}=\mathrm{GENID}(5,5,2, \mathrm{SQRT} \mid 3,1,-1) \mid \operatorname{ROSAD}(2,2 * \mathrm{SQRT} \mid 3 /$ $3,3,120) \mid[1, \mathrm{SQRT}|3 / 3,1 ; 3, \mathrm{SQRT}| 3 / 3,1]\}$

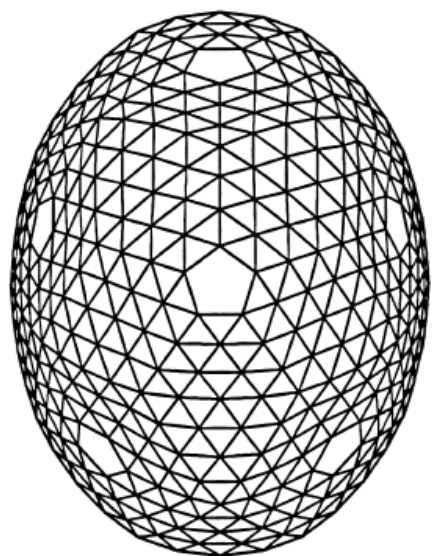

Fig. 48 View of a geodesic form.

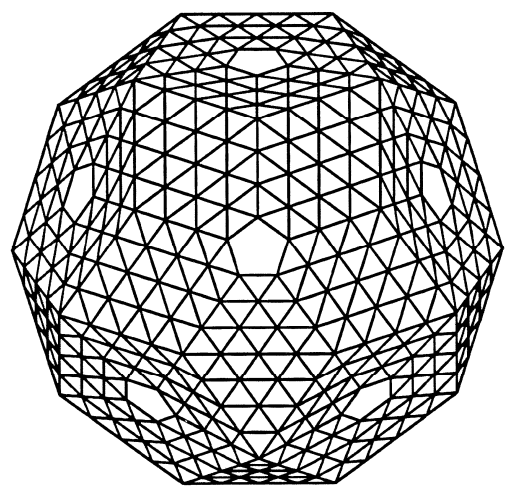

Fig. 49 View of an icosidodecahedral configuration.

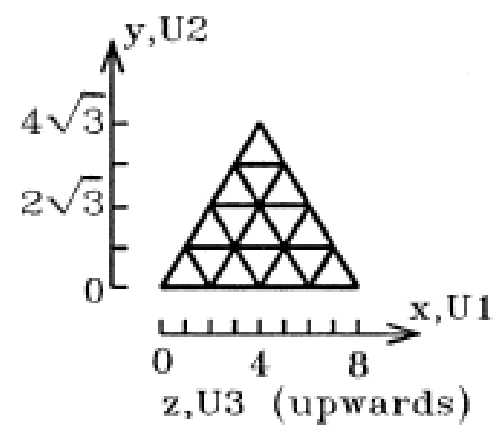

Fig. 50 A triangular form.

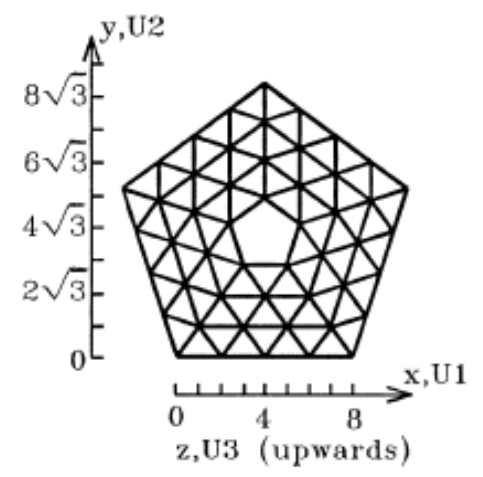

Fig. 51 A pentagonal form.

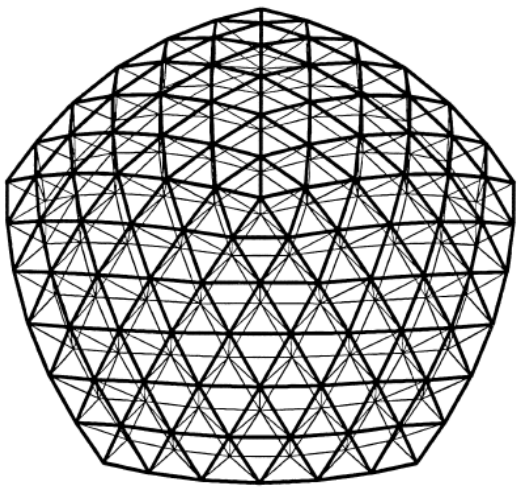

Fig. 52 A double layer geodesic form.

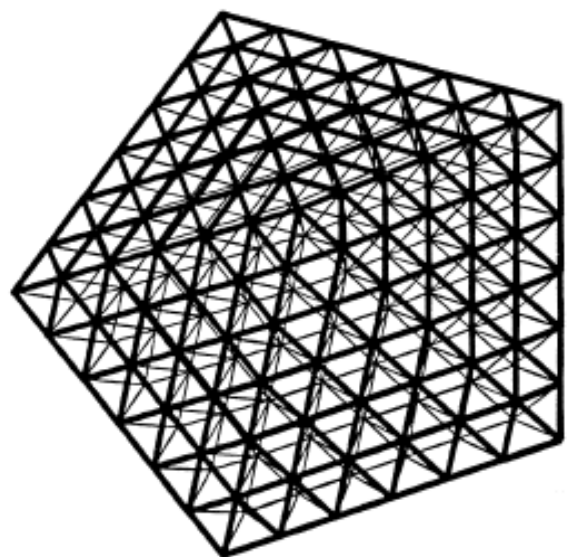

Fig. 53 A double layer polyhedric configuration.

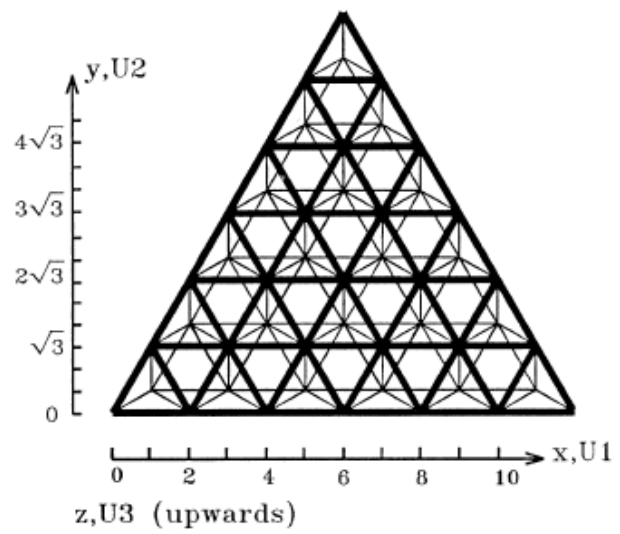

Fig. 54 A triangular double layer configuration. 
In Figs. 52-54 the top layer elements are drawn in thick lines and the bottom layer elements as well as the web elements are drawn in thin lines.

\section{Conclusions}

In For large and complex structural forms, the sheer volume of information to be handled can make data generation a time consuming and error prone task. To overcome this problem, suitable systems have been developed by which computer graphics and data generation for any type of structure can be done conveniently. Formex algebra is one such mathematical system. The ideas of formex algebra can be applied to many branches of science and technology. In this paper the concepts of formex algebra and its programming language Formian have been described in relation to a variety of polyhedric and geodesic configurations.

An important aspect of this paper is the establishment of the concepts and constructs through which polyhedric and geodesic configurations may be created with the polyhedron function. The polyhedron function provides a basis for the configuration processing of structural configurations in a compact and readily understood manner and it allows one to work with the same set of tools in all data generation problems eliminating the need for the employment of an assortment of programs which are dealing with specific problems.

\section{References}

[1] H. Nooshin and P. Disney, Formex Configuration Processing: Structural Morphology and Configuration Processing of Space Structures, Multi Science Publishers, London, 2009.

[2] B. Fuller and R. Marks, The Dymaxion World of Buckminster Fuller, AncorPress, 1973.

[3] D. Tzourmakliotou, Computer aided design of braced domes, PhD Thesis, University of Surrey, United Kingdom, 1993.

[4] A. Pugh, Polyhedra: A Visual Approach, University of California Press, 1976.

[5] R. Bury, Plato IX Timaeus, The Loeb Classical Library, Harvard University Press, 1975.

[6] H. Nooshin, P. Disney and O. Champion, Computer Aided Processing of Polyhedric Configuration: Beyond the Cube, The Architecture of Space Frames and Polyhedra, John Wiley \& Sons, New York, 1997, pp. 343-384.

[7] D. Tzourmakliotou, The polyhedric Configurations in Spatial Structures, in: Proceedings of the Sixth Conference on Computation of Shell and Spatial Structures, Spanning Nano to Mega, Iass-Iacm 2008, available online at: http://ecommons.library.cornell. edu.bitstream/1813/11539/StructuralMorphology.pdf, pp. 10-14.

[8] H. Nooshin and P. L. Disney, Formex configuration processing II, International Journal of Space Structures 16 (1) (2001) 1-56.

[9] H. Nooshin and P. L. Disney, Formex configuration processing III, International Journal of Space Structures 17 (1) (2002) 1-50.

[10] R. Motro, Review of the development of geodesic domes, in: Z. S. Makowski (ed.), Analysis, Design and Construction of Braces Domes, Granada publishing Ltd, London, 1984, pp. 387-411.

[11] A. Greorghiu and V. Dragomir, Geometry of Structural Forms, Elsevier applied science publishers, London, 1978. 